DESY 10-237

MPP-2010-166

\title{
Hunting Dark Matter Gamma-Ray Lines with the Fermi LAT
}

\author{
Gilles Vertongen ${ }^{1}$, Christoph Weniger ${ }^{2}$ \\ 1 Deutsches Elektronen-Synchrotron (DESY), Notkestrasse 85, 22603 Hamburg, Germany \\ 2 Max-Planck-Institut für Physik, Föhringer Ring 6, 80805 München, Germany
}

\begin{abstract}
Monochromatic photons could be produced in the annihilation or decay of dark matter particles. At high energies, the search for such line features in the cosmic gamma-ray spectrum is essentially background free because plausible astrophysical processes are not expected to produce such a signal. The observation of a gamma-ray line would hence be a 'smoking-gun' signature for dark matter, making the search for such signals particularly attractive. Among the different dark matter models predicting gamma-ray lines, the local supersymmetric extension of the standard model with small $R$-parity violation and gravitino LSP is of particular interest because it provides a framework where primordial nucleosynthesis, gravitino dark matter and thermal leptogenesis are naturally consistent. Using the two-years Fermi LAT data, we present a dedicated search for gamma-ray lines coming from dark matter annihilation or decay in the Galactic halo. Taking into account the full detector response, and using a binned profile likelihood method, we search for significant line features in the energy spectrum of the diffuse flux observed in different regions of the sky. No evidence for a line signal at the $5 \sigma$ level is found for photon energies between 1 and $300 \mathrm{GeV}$, and conservative bounds on dark matter decay rates and annihilation cross sections are presented. Implications for gravitino dark matter in presence of small $R$-parity violation are discussed, as well as the impact of our results on the prospect for seeing long-lived neutralinos or staus at the LHC.
\end{abstract}




\section{Introduction}

Gravitational evidences for dark matter exist from galactic to cosmological scales, but its particle nature still remains unknown [1 3 ]. The most popular dark matter candidate, a weakly interacting massive particle (WIMP), could in principle be detected directly through its scattering on atomic nuclei, indirectly by observing traces of its annihilation products in the cosmic-ray fluxes, or by its direct production at colliders. Since the stability of dark matter is by no means established, there is also the possibility to observe cosmic-ray contributions stemming from the decay of dark matter particles. However, despite lots of efforts, no unambiguous non-gravitational dark matter signal has been found so far.

Indirect searches for dark matter annihilation or decay products in the cosmic gammaray, anti-particle and neutrino fluxes are limited not only by the instrument sensitivities, but also by our understanding of the astrophysical foregrounds. One of the few dark matter signatures that would unambiguously stand out of the astrophysical foreground are the monochromatic photons potentially produced in two-body annihilation or decay of dark matter. They would appear as a line feature in the otherwise continuous gammaray energy spectrum [ 1 [ 6$]$. The observation of such a gamma-ray line would thus be of paramount interest for the understanding of dark matter in the Universe.

The production of gamma-ray lines is expected in many theoretical dark matter models. The corresponding branching ratios are often very small, but this could be compensated by the high experimental sensitivity to such signals. In the popular scenario where dark matter is the lightest neutralino $\chi_{1}^{0}$ of the minimal supersymmetric standard model (MSSM) [7], gamma-ray lines from $\chi_{1}^{0} \chi_{1}^{0} \rightarrow \gamma \gamma$ or $\chi_{1}^{0} \chi_{1}^{0} \rightarrow \gamma Z^{0}$ annihilations are only produced at the one-loop level [8-14], and they would be mostly out of reach for current experiments. However, their production can be enhanced in non-minimal variations of the MSSM [15, 16]. Alternatively, a large number of viable WIMP models exist, some of them predicting a sizable annihilation into monochromatic photons, e.g. singlet dark matter [17, hidden U(1) dark matter [18 20], effective dark matter scenarios [21], scenarios with strong annihilation into the higgs boson and a photon [22], inert higgs doublet dark matter [23], or Kaluza-Klein dark matter in models with universal extra dimensions [24, 25]. Additionally, prominent spectral features in the gamma-ray flux can be generated by the internal Bremsstrahlung or final state radiation potentially produced in dark matter annihilation [26 29].

An interesting example of decaying dark matter is the gravitino $\psi_{3 / 2}$, which appears in locally supersymmetric extensions of the Standard Model. In scenarios where $R$-parity and the lepton number are slightly violated and the gravitino is the lightest superparticle (LSP), thermal leptogenesis, gravitino dark matter and primordial nucleosynthesis are naturally consistent [30]. Within this framework, the gravitino is no longer stable and decays on cosmological time scales 31], thus rendering the imprints of its decay potentially observable in the cosmic-ray fluxes [32 43]. Interestingly, the line feature generated by the two-body decay into neutrinos and photons, $\psi_{3 / 2} \rightarrow \gamma \nu$, is prominent for a wide range of gravitino masses [33], suggesting that the first observation of gravitino dark matter could happen via gamma-ray lines.

In general, dark matter lifetimes of the order of $10^{26}-10^{29} \mathrm{~s}$, which is in the ballpark 
of what is accessible by cosmic-ray experiments in the $\mathrm{GeV}-\mathrm{TeV}$ regime, are obtained when the symmetry responsible for the dark matter stability is violated by dimension six operators generated close to the grand unification scale [44, 45]. A typical example, which has a large branching fraction into monochromatic photons, is the hidden $\mathrm{SU}(2)$ vector dark matter [46]. Note that even if dark matter only decays into charged leptons at tree level, like in the models designed to explain the PAMELA/Fermi LAT $e^{ \pm}$measurements [47 49], gamma-ray lines produced at the one-loop level could potentially be observable [50].

A dedicated search for Galactic gamma-ray lines with energies between 30 and $200 \mathrm{GeV}$ using the Fermi Large Area Telescope (LAT) data [51] has been presented in Ref. [52], where the all-sky averaged diffuse gamma-ray flux measured between 7 Aug 2008 and 21 Jul 2009 was considered. Searching for monochromatic features in the energy spectrum, their null result enable them to put constraints on the dark matter annihilation cross section and decay rate into gamma-ray lines. A similar analysis using the EGRET 1 data has been performed for gamma-ray line energies between 0.1 and $10 \mathrm{GeV}$ in Ref. [53].

The extra-galactic gamma-ray background (EGBG), as derived from the Fermi LAT data in Ref. [54], has also been used in Ref. 55] to put constraints on gamma-ray lines from cosmological dark matter annihilation [56, 57]. In that case, the redshifted gammaray lines lead to less pronounced features in the energy spectrum than the Galactic ones. The lack of spectral features in the EGBG was used to put limits on the corresponding annihilation cross section. Further gamma-line searches, coming from different observations, and ranging from energies of $10 \mathrm{keV}$ up to $10 \mathrm{TeV}$, were presented for annihilation in Ref. [58] and for decay in Ref. [59]. General strategies for the detection of gamma-ray lines from dark matter annihilation with Fermi LAT and future experiments were discussed in Refs. 60 62].

The purpose of this paper two-fold: First, we will update and extend the gamma-ray line analysis of the Fermi LAT data presented in Ref. [52] to a larger energy range of 1-300 GeV, searching for significant line signals that might come from dark matter annihilating or decaying in the Galactic dark matter halo, and derive conservative constraints. Although highly relevant for the gravitino dark matter scenario, no dedicated search for gamma-ray lines from decaying dark matter in the whole $\mathcal{O}(\mathrm{GeV}-\mathrm{TeV})$ energy regime has been performed so far. Furthermore, the large statistics of the Fermi LAT data allows us to further probe the low energy regime, i.e. energies from 1 to $10 \mathrm{GeV}$, and thus strengthening the previous EGRET bounds on annihilating dark matter [53]. Such energies are of particular interest in the view of the recent hints from direct dark matter detection reported by the CDMS 63], CoGeNT [64] and DAMA [65, 66] collaborations. Second, we will discuss the impact of our results on decaying gravitino dark matter. Covering previously unexplored values of the gravitino mass, we will use our limits on gamma-ray lines to constrain the size of $R$-parity violation, which can be translated into lower limits on the decay lengths of neutralino or stau NLSPs.

The paper is organized as follows: In section 2, we present our analysis of the Fermi LAT

\footnotetext{
${ }^{1}$ See http://heasarc.gsfc.nasa.gov/docs/cgro/cgro/egret.html.
} 
data. After briefly discussing the gamma-ray flux produced in the dark matter annihilation or decay, we present the strategy for data extraction and line searches in section 2.1. Section 2.2 is devoted to a presentation of the resulting bounds on annihilation cross section and decay rate, whereas in section 2.3 we discuss details and caveats of our analysis. In section 3 , we apply our results to gravitino dark matter and discuss prospects for NLSP observations at particle colliders. We then conclude in section 4.

\section{Gamma-ray line constraints from Fermi LAT}

Our line search is based on the measurements of the cosmic gamma-ray flux performed since August 2008 by the Large Area Telescope (LAT), the main instrument on the Fermi Gamma-ray Space Telescope. The LAT is a pair conversion detector, designed to observe gamma rays with energies ranging from $30 \mathrm{MeV}$ to more than $300 \mathrm{GeV}$ with an energy resolution of about $10 \%$. It has a large field-of-view that spans around $20 \%$ of the sky, allowing an effective measurement of the all-sky diffuse fluxes. From the four event classes presented in the available public data, we only consider the recently released 'DataClean' event class, which provides the best rejection of charged cosmic rays. This is especially important when analyzing the diffuse gamma-ray flux at high energies.

Gamma-ray lines may find their origin in the decay or annihilation of dark matter particles $\psi$ in the Galactic halo. The observed gamma-ray flux at Earth is given by a lineof-sight integral depending linearly on the dark matter distribution $\rho_{\mathrm{dm}}(r)$ when considering dark matter decays

$$
\frac{d J_{\gamma}}{d E}(\xi)=\frac{\Gamma_{\psi \rightarrow \gamma \nu}}{4 \pi m_{\psi}} \delta\left(E-\frac{m_{\psi}}{2}\right) \underbrace{\int_{\text {l.o.s. }} d s \rho_{\mathrm{dm}}(r)}_{\equiv J_{\rho}^{\text {dec. }}},
$$

while in the annihilation case, it depends quadratically on $\rho_{\mathrm{dm}}(r)$

$$
\frac{d J_{\gamma}}{d E}(\xi)=2 \frac{\langle\sigma v\rangle_{\psi \psi \rightarrow \gamma \gamma}}{8 \pi m_{\psi}^{2}} \delta\left(E-m_{\psi}\right) \underbrace{\int_{\text {l.o.s. }} d s \rho_{\mathrm{dm}}^{2}(r)}_{\equiv J_{\rho}^{\text {ann. }}} .
$$

Here, $m_{\psi}$ is the dark matter mass, while $\Gamma_{\psi \rightarrow \gamma \nu}$ and $\langle\sigma v\rangle_{\psi \psi \rightarrow \gamma \gamma}$ denote the dark matter decay width and annihilation cross section into gamma-ray lines, respectively. The coordinate $s$ runs along the line of sight, in a direction spanning an angle $\xi$ with respect to the Galactic center direction. The distance to the Galactic center $r$ is related to the distance to the Sun $s$ through $r(s, \xi)=\sqrt{\left(r_{0}-s \cos \xi\right)^{2}+(s \sin \xi)^{2}}$, where $r_{0}=8.5 \mathrm{kpc}$ is the distance of the Sun to the Galactic center [67, 68].

We consider the Navarro-Frenk-White (NFW) profile [52, 69]

$$
\rho_{\mathrm{dm}}(r)=\rho_{0}\left[\frac{r}{r_{0}}\right]^{-\gamma}\left[\frac{1+\left(r_{0} / r_{s}\right)^{\alpha}}{1+\left(r / r_{s}\right)^{\alpha}}\right]^{\frac{\beta-\gamma}{\alpha}}
$$




\begin{tabular}{cccc}
\hline Target region & Geometry & $\begin{array}{c}\int_{\Delta \Omega} d \Omega J_{\rho}^{\text {dec. }} \\
{\left[\mathrm{GeV} \mathrm{cm}^{-2} \mathrm{sr}\right]}\end{array}$ & $\begin{array}{c}\int_{\Delta \Omega} d \Omega J_{\rho}^{\text {ann. }} \\
{\left[\mathrm{GeV}^{2} \mathrm{~cm}^{-5} \mathrm{sr}\right]}\end{array}$ \\
\hline Halo & $|b| \geq 10^{\circ}$ & $2.2 \times 10^{23}$ & $8.3 \times 10^{22}$ \\
Center & $\begin{array}{c}|l| \leq 36^{\circ} \text { and } 5^{\circ} \leq|b| \leq 36^{\circ} \\
\text { plus }|l| \leq 7^{\circ} \text { and }|b| \leq 5^{\circ}\end{array}$ & $6.9 \times 10^{22}$ & $9.2 \times 10^{22}$ \\
\hline
\end{tabular}

Table 1: Geometry of our target regions in terms of Galactic longitude $l$ and latitude $b$.

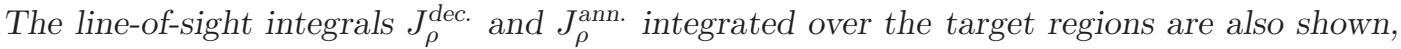
assuming a NFW profile.

which is defined by $(\alpha, \beta, \gamma)=(1,3,1)$ and $r_{s}=20 \mathrm{kpc}$, as a reference for the dark matter distribution. For the sake of comparison, we also consider the isothermal profile with $(\alpha, \beta, \gamma)=(2,2,0)$ and $r_{s}=3.5 \mathrm{kpc}$, as well as the Einasto profile [70 72 ]

$$
\rho_{\mathrm{dm}}(r)=\rho_{0} \exp \left[-\frac{2}{\alpha}\left(\frac{r^{\alpha}-r_{0}^{\alpha}}{r_{s}^{\alpha}}\right)\right] \quad \text { with } \quad \alpha=0.17, \quad r_{s}=20 \mathrm{kpc},
$$

which is favored by the latest $N$-body simulations.2 2 All profiles are normalized to $\rho_{\mathrm{dm}}(r=$ $\left.r_{0}\right)=0.4 \mathrm{GeV} \mathrm{cm}^{-3}$ at Sun's position [74, 75]. The resulting line-of-sight integrals $J_{\rho}^{\text {dec. }}$ and $J_{\rho}^{\text {ann. }}$, integrated over our target regions (see below), are summarized in Tab. 1 for the NFW profile. Note that substructures in the Galactic dark matter distribution can change the angular profile and magnitude of the gamma-ray emission (see e.g. Refs. [62, 72]). If this is the case, our results can straightforwardly be generalized by adopting the corresponding values for $J_{\rho}^{\text {dec./ann. }}$.

\subsection{Methods}

The gamma-ray events that enter our analysis are selected from the 'DataClean' event class of the Fermi LAT data measured between 4 Aug 2008 and 17 Nov 2010.3 From all events recorded by the Fermi LAT, we select energies between $100 \mathrm{MeV}$ and $500 \mathrm{GeV}$, and apply the zenith angle criterion $\theta<105^{\circ}$ in order to avoid contamination by the Earth's Albedo 4 Below $1 \mathrm{GeV}$, the fast variation of the effective area as well as the presence of small and sharp jumps in the energy spectrum (presumably related to side effects of the energy reconstruction [76, 77]) introduce spurious effects in our line search. Furthermore, the nominal energy range of the Fermi LAT ends at $300 \mathrm{GeV}$ [51]. As a consequence, we concentrate on gamma-ray line energies between 1 and $300 \mathrm{GeV}$. Systematic uncertainties,

\footnotetext{
${ }^{2}$ However, see Ref. [73] for observational arguments in favor of cored dark matter profiles.

${ }^{3}$ The event data, as well as the corresponding information about the instrument response functions concerning angular and energy resolution, are available at http://fermi.gsfc.nasa.gov/ssc/data/.

${ }^{4}$ These selections are made using the Fermi Science Tools v9r18p6, see http://fermi.gsfc.nasa.gov/ssc/data/analysis/software/. As cut in gtmktime we took DATA_QUAL==1 \&\& LAT_CONFIG==1 \&\& ABS (ROCK_ANGLE) $<52$.
} 

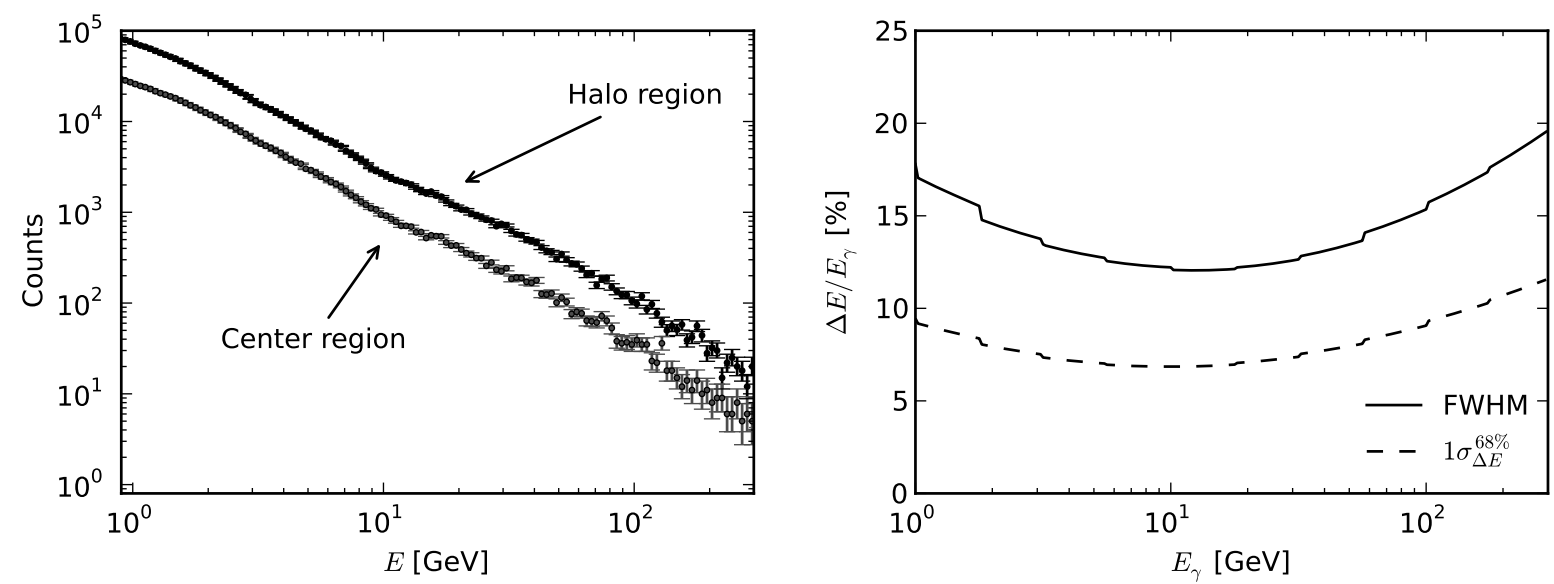

Figure 1: Left panel: Observed counts in the halo and the center regions, binned into 50 bins per energy decade (200 bins per decade are used in our likelihood analysis). Right panel: Fermi LAT energy resolution $\Delta E / E_{\gamma}$, in terms of FWHM and $68 \%$ containment $\sigma_{\Delta E}^{68 \%}$, as derived from the IRF of the 'DataClean' event class.

as well as limitations of the analysis method, are discussed in section 2.3 in light of the data.

When looking for dark matter signals in gamma-ray maps that cover the whole sky, it is critical to choose a target region which maximizes the corresponding signal-to-noise ratio $S / N$. The two regions that we found giving a very good $S / N$ for decaying dark matter ('halo region') or annihilating dark matter ('center region') are summarized in Tab. 1. Although optimized for the NFW profile, they also yield good $S / N$ for the Einasto and isothermal profiles.

Since the selection procedure leaves us with a large number of $1.3 \times 10^{6}\left(5 \times 10^{5}\right)$ events above $1 \mathrm{GeV}$ for the halo (center) region, we perform a binned analysis of the data. To this end, we distribute the events into 200 logarithmically equally spaced energy bins per decade, and sum over the angles. This gives a sequence of count numbers $c_{i} \in \mathbb{N}_{0}$, which is illustrated in the left panel of Fig. 1 for both target regions. Note that we do not perform a point source subtraction in this work. A proper treatment would mask out only $\mathcal{O}(5 \%)$ of the events [52], and hence only marginally affect our results.

The spectral feature produced by a gamma-ray line can be inferred from the Fermi LAT instrument response function (IRF). Its most recent version, 'Pass6 version 3', was determined using Monte Carlo generated samples of photon events between $18 \mathrm{MeV}$ and $562 \mathrm{GeV}$, and includes effects measured in-flight, see Refs. [78, 79]. It contains the pointspread-function (PSF), as well as the energy dispersion $\mathcal{D}\left(E, E_{\gamma}\right)$ which describes the distribution of the reconstructed energies $E$ as a function of the physical photon energy $E_{\gamma}$. In order to integrate out the implicit dependence of the energy dispersion on the event impact angle with respect to the detector axis, $\mathcal{D}\left(E, E_{\gamma}\right)$ is averaged over this impact angle weighted by its distribution in our data sample. The resulting full width at half maximum (FWHM) of the energy dispersion is shown in the right panel of Fig. 1, where the $68 \%$ 

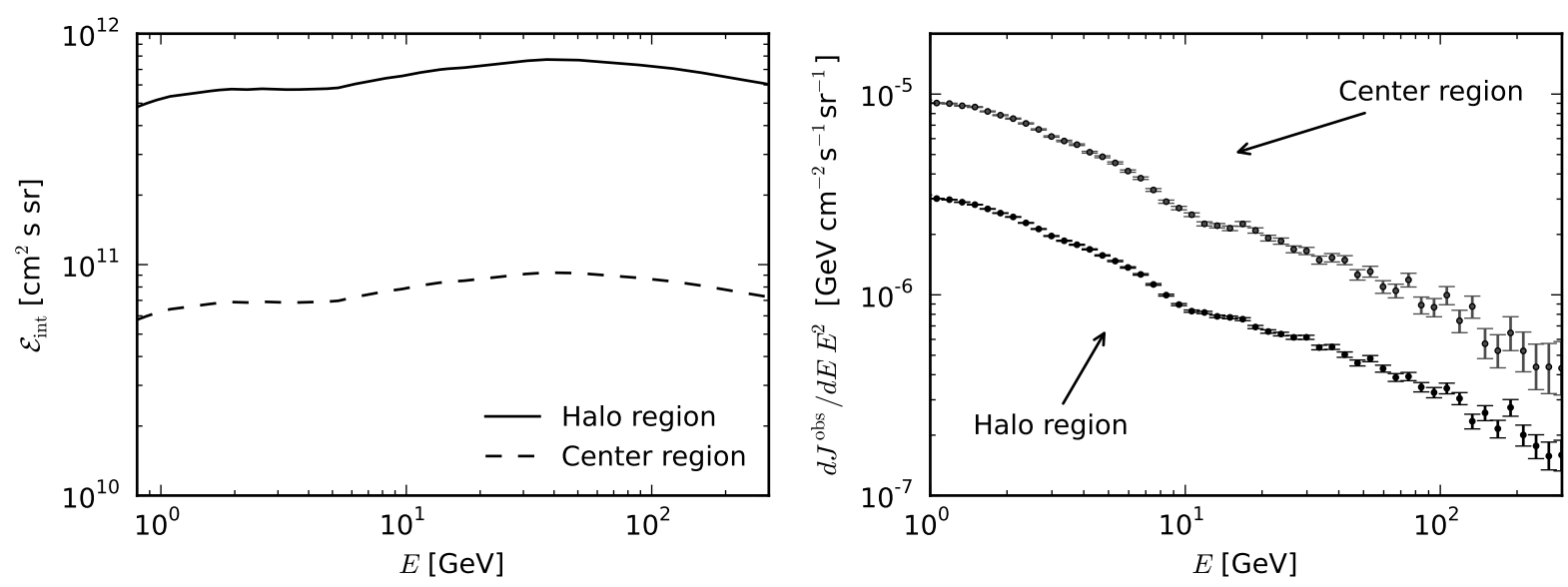

Figure 2: Left panel: Integrated exposures $\mathcal{E}_{\text {int }}$ for both target regions, following the weighting method described in the text. Right panel: Differential gamma-ray flux as observed in the two target regions.

containment energy resolution $\sigma_{\Delta E}^{68 \%}$ is also shown 5 Lastly, since our two samples have a large sky coverage, the effects of the PSF, which features a $68 \%$-containment angle well below $1^{\circ}$ at energies above $1 \mathrm{GeV}$, can be safely neglected.

The profile likelihood method [80, 81] is used to calculate the significance of a potential gamma-ray line contribution to the observed gamma-ray flux. The likelihood function is defined as

$$
L(\boldsymbol{\mu} \mid \mathbf{c})=\prod_{i=i_{0}, \ldots, i_{1}} P_{\mu_{i}}\left(c_{i}\right),
$$

where, for each energy bin $i, \mu_{i}$ and $c_{i}$ denote the expected and observed count numbers, respectively, and $P_{\mu}(k)=\mu^{k} e^{-\mu} / k$ ! is the Poisson probability mass function with mean value $\mu$. The data are modeled by a simple power law with a spectral index $-\gamma$ and a normalization $\beta$, plus a line signal at fixed energy $E_{\gamma}$ with the normalization $\alpha$. The power law background is supposed to take into account not only the gamma-ray flux of astrophysical origin, but also the gamma-ray continuum which might be produced in the dark matter annihilation or decay additionally to the sharp gamma-ray line. Since the power law is only locally a good approximation to the background fluxes, we use a sliding energy window in the fitting procedure. For gamma-ray energies $E_{\gamma} \lesssim 10 \mathrm{GeV}$ we only take into account the energy bins $i \in\left[i_{0}, i_{1}\right]$ that lie within $\pm 2 \sigma_{\Delta E}^{68 \%}$ around $E_{\gamma}$, whereas, due to limited statistics, at high energies $E_{\gamma} \gtrsim 100 \mathrm{GeV}$ bins within a wider energy window from roughly $\frac{1}{3} E_{\gamma}$ to $3 E_{\gamma}$ are considered. In the range $10 \mathrm{GeV} \lesssim E_{\gamma} \lesssim 100 \mathrm{GeV}$ the energy window size is interpolated between these two cases. Furthermore, events above $300 \mathrm{GeV}$ are always excluded from the analysis, which leads to energy windows asymmetric around

\footnotetext{
${ }^{5}$ Our resulting FWHM is larger than the one discussed in Ref. [52] by a factor of 1.1-1.4, which presumably affects our limits at the level of $5 \%-20 \%$. The difference is probably due to different energy reconstruction methods underlying the public 'DataClean' event class used in this work and the dedicated analysis in Ref. [52]. However, the $68 \%$ containment agrees well with Ref. [51].
} 
the highest gamma-ray line energies. The expected number of counts in each energy bin is given by

$$
\mu_{i}(\alpha, \beta, \gamma)=\int_{E_{i}^{-}}^{E_{i}^{+}} d E^{\prime}\left(\beta \mathcal{E}_{\text {int }}\left(E^{\prime}\right) E^{\prime-\gamma}+\alpha \mathcal{E}_{\text {int }}\left(E_{\gamma}\right) \mathcal{D}\left(E^{\prime}, E_{\gamma}\right)\right)
$$

where the $E_{i}^{ \pm}$denote the boundaries of each energy bin $i, \mathcal{E}_{\text {int }}$ is the energy dependent integrated total exposure, and we neglect the effects of the energy dispersion on the powerlaw background flux. To obtain $\mathcal{E}_{\text {int }}$, we integrate the exposure maps, which we derived with the Fermi Science Tools, 6 over the observed region of the sky, weighted by the angular profile of the expected gamma-ray line signal from dark matter decaying or annihilating inside the Galactic halo. The resulting integrated exposures are practically identical for decaying and annihilating dark matter signals, and are shown in the left panel of Fig. 2 for the halo and center regions. They can be approximated by $\mathcal{E}_{\text {int }}(E) \simeq\langle\mathcal{E}(E)\rangle \Delta \Omega$, where the target region averaged exposures are in the range $\langle\mathcal{E}(E)\rangle \simeq 5-8 \times 10^{10} \mathrm{~cm}^{2} \mathrm{~s}$, and $\Delta \Omega=10.4 \mathrm{sr}(1.30 \mathrm{sr})$ is the solid angle spanned by the halo (center) region. The observed differential gamma-ray fluxes for both target regions, as inferred from the exposure and count maps, are shown in the right panel of Fig. 2.

Depending on whether the normalization $\alpha$ of the gamma-ray line is kept free or fixed, the likelihood function in Eq. (5) must be maximized with respect to three or two parameters, respectively:

$$
L(\mathbf{c})=\sup \left\{L(\boldsymbol{\mu} \mid \mathbf{c}): \alpha, \beta, \gamma \in \mathbb{R}^{+}\right\} \quad \text { or } \quad L_{\alpha}(\mathbf{c})=\sup \left\{L(\boldsymbol{\mu} \mid \mathbf{c}): \beta, \gamma \in \mathbb{R}^{+}\right\} .
$$

Defining $\Lambda_{\alpha}(\mathbf{c}) \equiv L_{\alpha}(\mathbf{c}) / L(\mathbf{c})$, the $95 \%$ C.L. interval upper limit of the gamma-ray line normalization is given by the largest value of $\alpha$ which satisfies $-2 \log \Lambda_{\alpha}(\mathbf{c}) \leq 4$. The parameter $\alpha$ is defined such that it can be directly identified with the corresponding gammaray line flux, i.e. $J_{\gamma} \equiv \alpha$. In the absence of a gamma-ray line signal at the $5 \sigma$ level, which would correspond to $-2 \log \Lambda_{\alpha=0}(\mathbf{c}) \geq 23.7$, we quote the upper limit of the $95 \%$ C.L. interval as the upper limit on the gamma-ray line flux. Note that the $-2 \log \Lambda_{\alpha=0}$ value corresponding to a $5 \sigma$-confidence level slightly deviates from the naively expected 25 , because the normalization of the gamma-ray line is bounded from below, i.e. $\alpha \geq 0$. The adopted value can be derived by recognizing that in absence of a gamma-ray line in $50 \%$ of the cases the optimal fit would actually require an unphysical negative normalization of the gamma-ray line, since for large enough count numbers statistical fluctuations are symmetric around their mean. These cases yield $-2 \log \Lambda_{\alpha=0}=0$, whereas in the other $50 \%$ of the cases, the $-2 \log \Lambda_{\alpha=0}$ would follow a conventional $\chi^{2}$-distribution with one degree of freedom 7

\footnotetext{
${ }^{6}$ In gtltcube we use a zenith cut zmax=105 to properly calculate the exposure also in cases where the field-of-view of the satellite intersects with the Earth's Albedo. However, due to our above cut on the satellite rocking angle (see footnote 4) this has only a small effect on the total exposure.

${ }^{7}$ We confirmed this behavior with a Monte Carlo analysis. Details about the likelihood-ratio and confidence intervals can be found e.g. in Refs. [82, 83]. Note that we do not use a "trial factor" as discussed in Refs. [76, 84], which only becomes relevant when constraining models that predict a multitude of lines or when claiming a line discovery.
} 


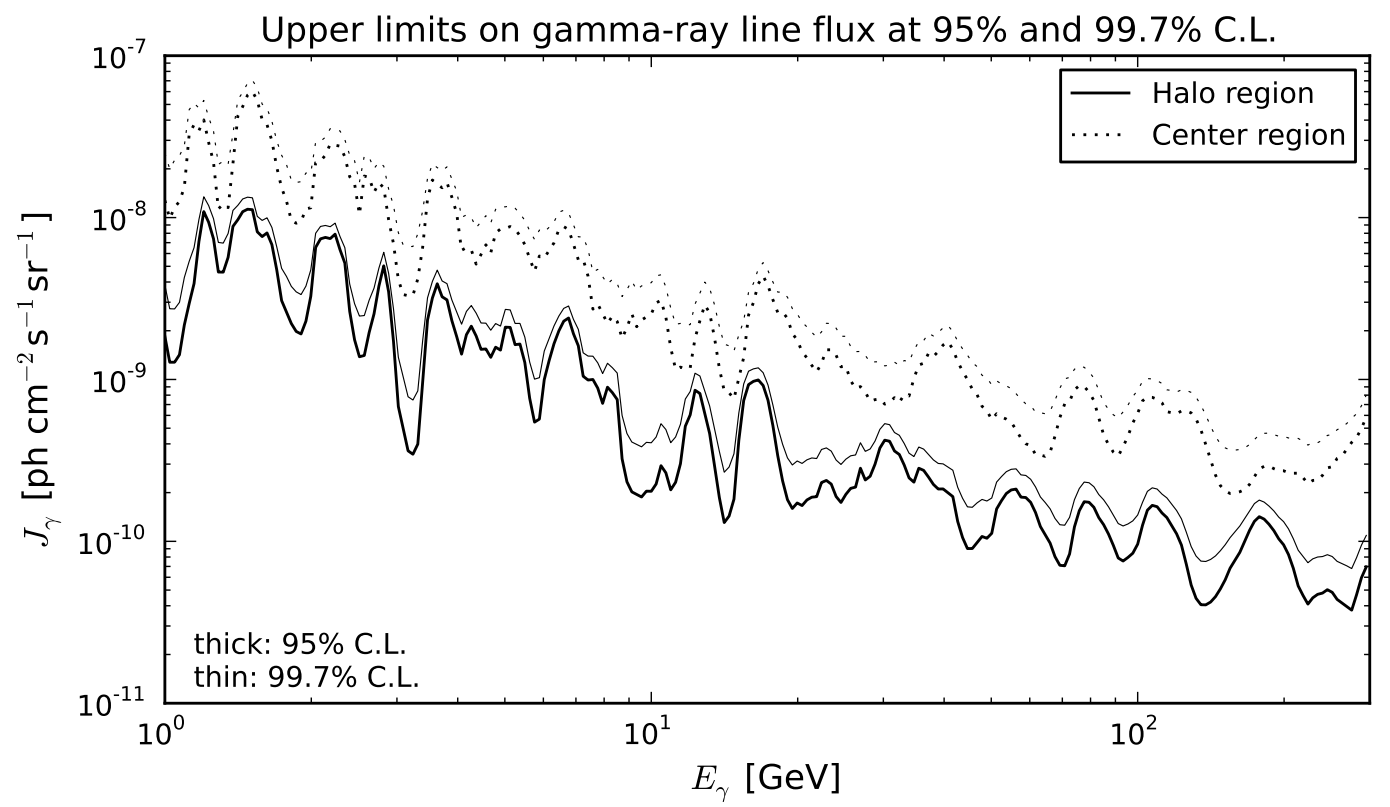

Figure 3: Our results for the $95 \%$ and $99.7 \%$ C.L. upper limits on the monochromatic photon flux from the halo and the center regions, as function of the gamma-ray line energy $E_{\gamma}$. Note that due to instrumental effects these limits are expected to be too strong at lower energies in some cases (cf. section 2.3). The corresponding conservative limits are show in Tab. 3 (as well as in the Figs. 4 and 5 ).

The likelihood analysis is performed using PyFITS8 and PyMinuit, an interface to the function minimizer MINUIT 1.7.9.9

\subsection{Results}

No gamma-ray lines with $5 \sigma$ significance are found in the $1-300 \mathrm{GeV}$ energy range. Details and caveats of the analysis are discussed in section 2.3. Our results for the $95 \%$ and $99.7 \%$ C.L. limits on the gamma-ray line flux are shown in Fig. 3 as a function of the gamma-ray line energy for both halo and center regions. As discussed in section 2.3, instrumental effects forbid us to take these limits at face value; the conservative limits summarized in Tab. 3 should be used instead. These are derived by quoting the weakest limit we obtain in given energy bands. Together with Eqs. (11) and (2), it is then straightforward to derive the corresponding constraints on the dark matter decay width or annihilation cross section.

Limits on the inverse dark matter decay width into monochromatic photons and neutrinos, $\Gamma_{\psi \rightarrow \gamma \nu}^{-1}$, as derived from the halo region observations, are presented in Fig. 4, assuming a NFW dark matter profile. The gray line shows the bounds as they directly follow from the flux limits in Fig. 3. In most of the considered energy range, they fluctuate around $10^{29} \mathrm{~s}$.

\footnotetext{
${ }^{8}$ PyFITS is a product of the Space Telescope Science Institute, which is operated by AURA for NASA, see http://www.stsci.edu/resources/software_hardware/pyfits

${ }^{9}$ See http://code.google.com/p/pyminuit/and/http://seal.web.cern.ch/seal/snapshot/work-packages/mathl
} 


\begin{tabular}{ccccccccc}
\hline \multirow{2}{*}{ Target region } & \multicolumn{3}{c}{$\Gamma_{\psi \rightarrow \gamma \nu}^{\text {profile }} / \Gamma_{\psi \rightarrow \gamma \nu}$} & & \multicolumn{3}{c}{$\langle\sigma v\rangle_{\psi \psi \rightarrow \gamma \gamma}^{\text {profile }} /\langle\sigma v\rangle_{\psi \psi \rightarrow \gamma \gamma}$} \\
\cline { 2 - 3 } & Iso. & NFW & Ein. & & Iso. & NFW & Ein. \\
\hline Halo & 0.96 & 1.00 & 1.00 & & 1.03 & 1.00 & 0.89 \\
Center & 1.03 & 1.00 & 0.91 & & 1.65 & 1.00 & 0.64 \\
\hline
\end{tabular}

Table 2: Rescaling factors for the bounds on the dark matter decay width and annihilation cross section (shown in Tab. 3), corresponding to the different halo profiles. In most cases, the weakest bounds are obtained for the isothermal profile (Iso.), the strongest bounds for the Einasto profile (Ein.), whereas the NFW profile leads to intermediate constraints.

The slight improvement with respect to the previous Fermi LAT limits [52] (dashed line) is due to the increased statistics after two years of data taking. The black dots represent the weakest limits obtained when varying the gamma-ray line energy within different adopted energy bands. Except at the highest energies, these values fluctuate around $6 \times 10^{28} \mathrm{~s}$, and are summarized in Tab. 3. Adopting halo profiles different from the NFW profile would change the limits by a few percent. The corresponding rescaling factors for the Einasto and the isothermal profiles are summarized in Tab. 2, and their impact on the limits is illustrated by the blue band in Fig. 4. Note that the normalization of the dark matter profile at Sun's position is another source of uncertainty, which is not captured in the rescaling parameters of Tab. 2. We do not plot the somewhat weaker limits on the decay width obtained from the center region, which can be found in Tab. 3 for completeness.

In Fig. 5 we present the limits on the dark matter annihilation cross section into gammaray pairs, $\langle\sigma v\rangle_{\psi \psi \rightarrow \gamma \gamma}$, as derived from the center region observations. As illustrated by the blue band, the uncertainties coming from the dark matter density profile are much larger than in the case of dark matter decay and of the order of $\sim 50 \%, c f$. Tab. 2, For comparison, we also show the previous limits obtained by EGRET [53] 10 The present analysis improves these limits by up to an order of magnitude, which is essentially due to the increased statistics of Fermi LAT with respect to EGRET. Furthermore, at high energies, we slightly strengthen the previous Fermi LAT constraints [52]. Our results are summarized in Tab. 3. It is interesting to note that, assuming the NFW profile, the limits on the dark matter annihilation cross section derived from the halo region are only a factor $\sim 2$ weaker than the limits derived from the center region.

Using kinematic considerations, our results can be straightforwardly translated into constraints on two-body decay or annihilation into a monochromatic photon plus a massive neutral particle $N$ with mass $m_{N}$. This is relevant for the common case of annihilation/decay into $\gamma Z^{0}$. However, in principle $m_{N}$ is a free parameter, and if $m_{N}$ is close to the dark matter mass $m_{\psi}$, the line signal can be even strongly enhanced in some scenarios [50].

\footnotetext{
${ }^{10}$ Note that the cross section limits shown in Fig. 8 of Ref. [53] are incorrectly too strong by a factor of ten, whereas the flux limits presented in Fig. 7 are correct [priv. comm. with the authors]. The EGRET cross section limits that we present in Fig. 5 are derived from Fig. 7 of Ref. [53].
} 


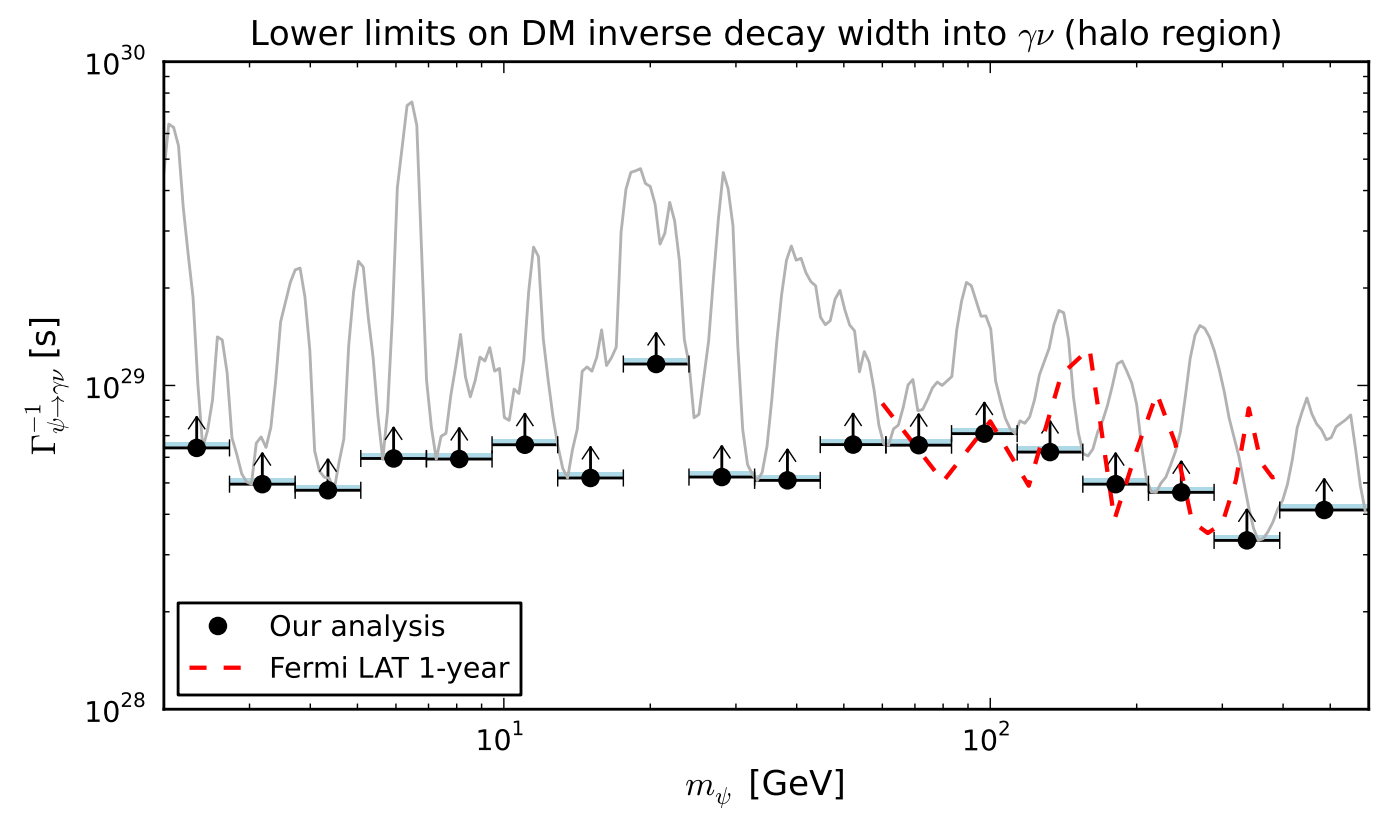

Figure 4: Lower bounds on the dark matter inverse decay width into monochromatic photons and neutrinos, $\Gamma_{\psi \rightarrow \gamma \nu}^{-1}$, as a function of the dark matter mass $m_{\psi}$, derived from the halo region fluxes assuming the NFW dark matter profile. The gray-solid line shows the 95\% C.L. limits as directly derived from the line flux limits shown in Fig. 3. The black dots show the weakest limits obtained in the adopted energy bands and are listed in Tab. 3 For comparison, the previous Fermi LAT limits from Ref. [52] are also shown by the red-dashed line (rescaled to our decay channel). The barely visible blue bands illustrate how the bounds change when using the isothermal or Einasto dark matter profiles instead.

Defining the quantity

$$
x \equiv 1+\sqrt{1+\frac{m_{N}^{2}}{E_{\gamma}^{2}}},
$$

which is bounded from below by $x \geq 2$, our limits for the decay into $\gamma \nu$ translate into limits on the decay into $\gamma N$ via

$$
\Gamma_{\psi \rightarrow \gamma N}\left(m_{\psi}\right)=\frac{x}{2} \Gamma_{\psi \rightarrow \gamma \nu}\left(E_{\gamma}\right), \quad \text { where } \quad m_{\psi}=x E_{\gamma} .
$$

For the annihilation scenario the cross section limits on $\psi \psi \rightarrow \gamma N$ are obtained by

$$
\langle\sigma v\rangle_{\psi \psi \rightarrow \gamma N}\left(m_{\psi}\right)=\frac{x^{2}}{2}\langle\sigma v\rangle_{\psi \psi \rightarrow \gamma \gamma}\left(E_{\gamma}\right), \quad \text { where } \quad m_{\psi}=\frac{x}{2} E_{\gamma} .
$$

Models that are partially constrained by our limits include singlet dark matter [17] and $\mathrm{SU}(2)$ vector dark matter [46], but also Refs. [19 22, 50]. Scenarios that remain practically unconstrained include the standard MSSM scenario [9], but also Refs. [15, 16, 23 25]. The particular case of gravitino dark matter will be discussed in the next section. 


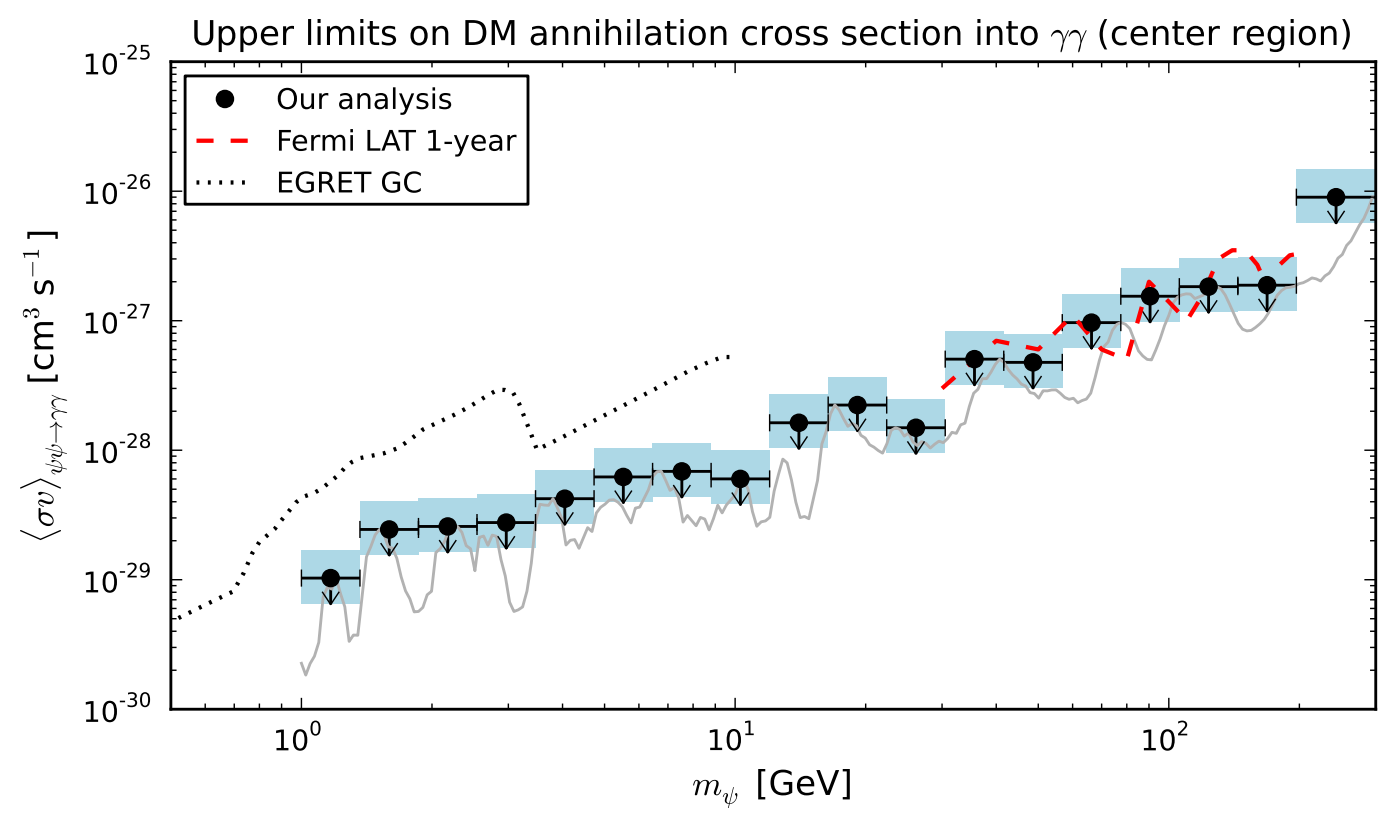

Figure 5: Upper bounds on the annihilation cross section into gamma-pairs, $\langle\sigma v\rangle_{\psi \psi \rightarrow \gamma \gamma}$, as a function of the dark matter mass $m_{\psi}$, derived from the center region fluxes assuming the NFW dark matter profile. The gray-solid line shows the 95\% C.L. limits as directly derived from the line flux limits shown in Fig. 3. The black dots show the weakest limits obtained in the adopted energy bands and are listed in Tab. 3. For comparison, the previous Fermi LAT limits from Ref. [52] as well as the limits derived from EGRET observations of the Galactic center [53] are also shown by the red-dashed and the black-dotted lines, respectively. The blue bands illustrate how the bounds change when using the isothermal or Einasto dark matter profiles instead.

\subsection{Discussion}

One crucial assumption underlying our analysis is that the background flux in the different considered energy windows can be well approximated by a power-law. This assumption is most likely to break down in cases where the statistics is very good. In order to check the validity of a power-law ansatz, we show in Fig. 6 the $\chi^{2} /$ d.o.f. of the background-only (green lines) and of the background-plus-signal (red lines) fits, as function of the gammaray line energy 11 The grey band corresponds to a $p$-value of $\geq 5 \%$. For the center region the fits are essentially in agreement with the data over the whole energy range. However, p-values significantly smaller than $5 \%$ occur at energies between 1 and $10 \mathrm{GeV}$ (as well as at high energies close to $300 \mathrm{GeV}$ ) when considering the halo region, which has a three times larger statistics than the center region. Assuming that the astrophysical gammaray fluxes follow smooth bended power-laws, this tension points to an instrumental effect, presumably related to the energy reconstruction of gamma-ray events.

\footnotetext{
${ }^{11}$ The smallness of the differences between the $\chi^{2} /$ d.o.f. of the background-plus-signal and backgroundonly fit at high energies comes from the fact that the $\chi^{2}$ values are actually dominated by the background and not by the narrow signal.
} 

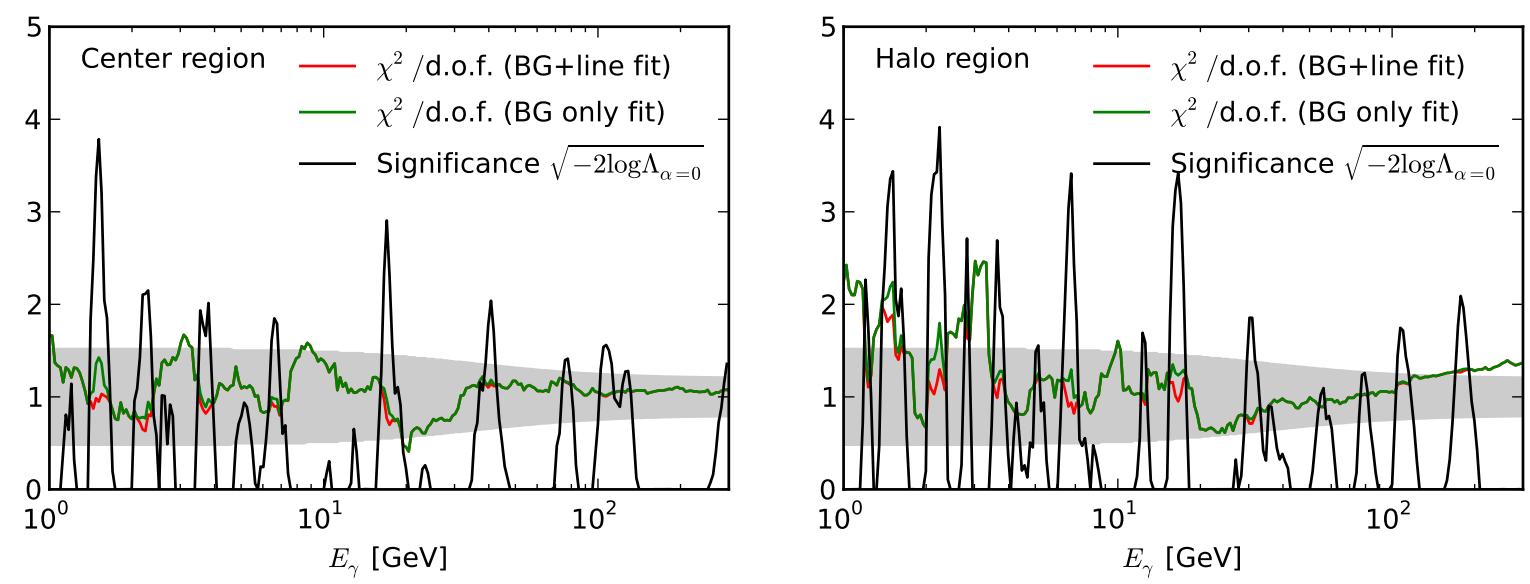

Figure 6: Significance of a potential line signal (black line) in the center (left panel) and halo (right panel) region in terms of standard deviations. We also show the $\chi^{2} /$ d.o.f. values of fits with (red line) and without (green line) line signal. The gray shaded area corresponds to a $p$-values of $\geq 5 \%$. Especially at low energies in the halo region, significant deviations occur from what is statistically expected in absence of a signal, as discussed in section 2.3 .

The black lines in Fig. [6 show the significance (in terms of standard deviations) of line signals with respect to a power-law background. Over the whole energy range we are performing $\mathcal{O}(50)$ statistically independent tests for a line. Hence, we expect to see a few cases with $2 \sigma$ significance, and practically no case with $3 \sigma$ significance. This expectation is marginally compatible with the observations of the center region, and is clearly violated for the halo region, where four $>3 \sigma$ excesses are observed below $20 \mathrm{GeV}$. However, an interpretation of these excesses in terms of gamma-ray lines would be suspicious: They occur at low energies in the halo region, where the statistics is best and hence the impact of instrumental effects most relevant, as indeed indicated by the bad $\chi^{2} /$ d.o.f. of the background-fits at this energy range. Moreover, most line-like features appear, although with a different significance, at the same position in both, the halo and the center region, indicating again a systematic effect. Without further understanding and reduction of instrumental effects, no conclusive statement about the existence of gamma-ray lines (below $\sim 5 \sigma$ significance) in this energy range can be made.

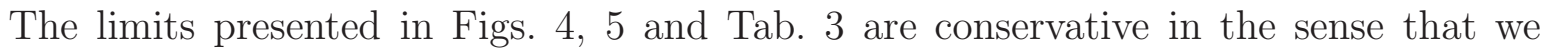
always quote the weakest limit we obtain in given energy bands. Especially in the halo region at energies below $20 \mathrm{GeV}$, this ensures that our limits are dominated (and weakened) by the presence of the line-like artefacts in the energy spectrum. A future removal of these artefacts from the data is expected to lead in general to a strengthening of the presented limits. Note that the presence of a real gamma-ray line signal significantly stronger than the artefacts would have lead to limits dominated by the line signal itself.

For two different gamma-ray line energies and for both center and halo regions, we show in Fig. 7 the line signals that are excluded at 95\% C.L. (for which $-2 \log \Lambda_{\alpha}(\mathbf{c})=4$, see above), and compare them with the best-fit background-only fluxes. The gamma-ray line energies are chosen such that the corresponding limits dominate in their respective 

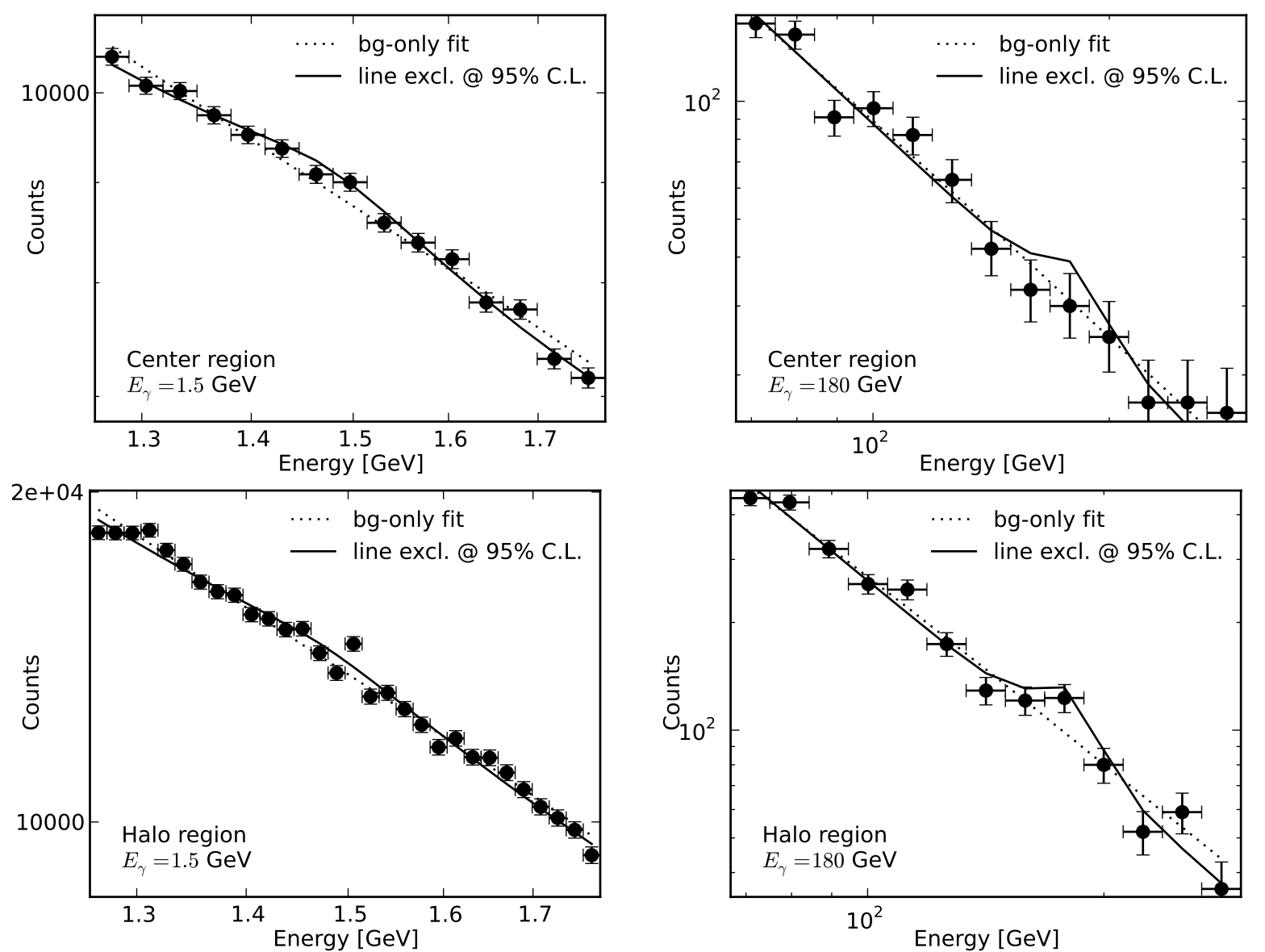

Figure 7: Line signals that are excluded at 95\% C.L. (solid line), compared to best-fit background-only model (dotted line) and data. We show results for the center (top panels) and halo (bottom panels) region. The gamma-ray line energies are $E_{\gamma}=1.5 \mathrm{GeV}$ (left panels) and $E_{\gamma}=180 \mathrm{GeV}$ (right panels). In the left panels, the signal-to-background ratio is $2.6 \%$ (top) and $1.5 \%$ (bottom).

energy bands in case of the halo region. Furthermore, the energy ranges shown in the plots correspond to the energy windows used in the fitting procedure, and we rebinned the data for better visibility. At low energies the gamma-ray line constraints essentially come from the non-observation of a strong bending in the measured energy spectrum (with signal-tobackground ratios as small as 1.5\%), whereas at high energies, where the considered energy window is larger, the constraints come from the non-observation of a well defined bump.

In summary, due to the apparent presence of line-like artefacts at the few percent level in the energy spectrum of the public 'DataClean' event class of the Fermi LAT, the flux limits from Fig. 3 are expected to be too strong in some cases, especially at energies below $\sim 20 \mathrm{GeV}$. Instead, the limits presented in Fig. [4, 5] and Tab. [3 should be used.

Further more general uncertainties and systematic effects enter our analysis [76, 77]: The systematic uncertainties in the effective area are energy dependent and increase from $5 \%$ at $560 \mathrm{MeV}$ to $20 \%$ at $10-100 \mathrm{GeV}$ in case of the 'DataClean' event class [54] (note that 


\begin{tabular}{|c|c|c|c|c|c|c|}
\hline \multirow[t]{2}{*}{$\overline{E_{\gamma}[\mathrm{GeV}]}$} & \multicolumn{2}{|c|}{$J_{\gamma}\left[\mathrm{ph} /\left(\mathrm{cm}^{2} \mathrm{~s} \mathrm{sr}\right)\right]$} & \multicolumn{2}{|c|}{$\Gamma_{\psi \rightarrow \gamma \nu}^{-1}[\mathrm{~S}]$} & \multicolumn{2}{|c|}{$\langle\sigma v\rangle_{\psi \psi \rightarrow \gamma \gamma}\left[\mathrm{cm}^{3} \mathrm{~s}^{-1}\right]$} \\
\hline & $\begin{array}{c}\text { halo } \\
\times 10^{-9}\end{array}$ & $\begin{array}{l}\text { center } \\
\times 10^{-9}\end{array}$ & $\begin{array}{l}\text { halo } \\
\times 10^{28}\end{array}$ & $\begin{array}{l}\text { center } \\
\times 10^{28}\end{array}$ & $\begin{array}{c}\text { halo } \\
\times 10^{-28}\end{array}$ & $\begin{array}{l}\text { center } \\
\times 10^{-28}\end{array}$ \\
\hline $1.0-1.4$ & 11 & 40 & 6.4 & 4.4 & 0.25 & 0.10 \\
\hline $1.4-1.9$ & 11 & 60 & 5.0 & 2.3 & 0.41 & 0.24 \\
\hline $1.9-2.5$ & 7.9 & 29 & 4.7 & 3.3 & 0.63 & 0.26 \\
\hline $2.5-3.5$ & 5.0 & 18 & 5.9 & 4.6 & 0.63 & 0.22 \\
\hline $3.5-4.7$ & 3.9 & 17 & 5.9 & 3.4 & 0.82 & 0.42 \\
\hline $4.7-6.5$ & 2.1 & 8.8 & 6.6 & 4.7 & 1.3 & 0.49 \\
\hline $6.5-8.8$ & 2.4 & 8.8 & 5.2 & 3.6 & 1.7 & 0.69 \\
\hline $8.8-12$ & 0.60 & 3.1 & 12 & 6.6 & 1.4 & 0.60 \\
\hline $12-16$ & 0.98 & 3.0 & 5.2 & 4.4 & 4.2 & 1.4 \\
\hline $16-22$ & 0.99 & 4.4 & 5.1 & 2.9 & 4.4 & 2.2 \\
\hline $22-31$ & 0.42 & 1.5 & 6.6 & 5.9 & 6.2 & 1.5 \\
\hline $31-42$ & 0.42 & 1.7 & 6.5 & 3.0 & 6.3 & 5.1 \\
\hline $42-57$ & 0.21 & 1.5 & 7.1 & 3.3 & 11 & 4.8 \\
\hline $57-77$ & 0.20 & 0.92 & 6.2 & 3.0 & 17 & 9.4 \\
\hline $77-106$ & 0.18 & 0.90 & 5.0 & 2.6 & 29 & 15 \\
\hline $106-144$ & 0.17 & 0.78 & 4.7 & 2.6 & 31 & 18 \\
\hline $144-197$ & 0.14 & 0.29 & 3.3 & 3.9 & 72 & 18 \\
\hline 197-300 & 0.10 & 0.59 & 4.1 & 1.2 & 96 & 91 \\
\hline
\end{tabular}

Table 3: Conservative upper limits on the flux in gamma-ray lines $J_{\gamma}$, as well as on the decay width $\Gamma_{\psi \rightarrow \gamma \nu}$ and the annihilation cross section $\langle\sigma v\rangle_{\psi \psi \rightarrow \gamma \gamma}$ of dark matter into monochromatic photons, for line energies $E_{\gamma}=1-300 \mathrm{GeV}$. In each of the indicated energy bands, we varied $E_{\gamma}$ to find the weakest possible $95 \%$ C.L. limit. The flux limits for all values of $E_{\gamma}$ are shown in Fig. 3 (see also section 2.3). The limits are evaluated for the halo and for the center region separately, and hold for the NFW profile. Other dark matter profiles just require a rescaling of the bounds as shown in Tab. 2.

these uncertainties amount mainly to an overall shift in the effective area). Uncertainties on the level of around $20 \%$ are expected to continue up to energies of hundreds of $\mathrm{GeV}$ [76, 77], but one has to keep in mind that a precise determination of the effective area at these high energies is still lacking due to limited statistics. However, even values reasonably larger than $20 \%$ would only mildly affect our conclusions. Over the whole considered energy range, the systematics of the LAT effective area consequently translate into uncertainties for our dark matter limits that range between $5 \%$ and $\sim 20 \%$. The absolute energy scale of the Fermi LAT is determined up to ${ }_{-10 \%}^{+5 \%}$ [48]. This translates into an additional uncertainty on the dark matter annihilation cross section $\langle\sigma v\rangle_{\psi \psi \rightarrow \gamma \gamma}$ of ${ }_{-20 \%}^{+10 \%}$, and on the decay width $\Gamma_{\psi \rightarrow \gamma \nu}$ of ${ }_{-10 \%}^{+5 \%}$, respectively.

Finally, we have also checked that when extending our analysis to $500 \mathrm{GeV}$ no significant line signals at high energies are revealed, and the limits below the highest energy band 
presented in Tab. 3 are only marginally affected. Considering the 197-300 GeV (300$500 \mathrm{GeV})$ energy band, we obtain in this case $\Gamma_{\psi \rightarrow \gamma \nu}^{-1}>6.0 \times 10^{28} \mathrm{~s}\left(1.7 \times 10^{28} \mathrm{~s}\right)$ for the halo region, and $\langle\sigma v\rangle_{\psi \psi \rightarrow \gamma \gamma}<4.1 \times 10^{-27} \mathrm{~cm}^{3} \mathrm{~s}^{-1}\left(1.54 \times 10^{-26} \mathrm{~cm}^{3} \mathrm{~s}^{-1}\right)$ for the center region. However, we emphasize that energies above $300 \mathrm{GeV}$ lie outside of the nominal energy range of the Fermi LAT.

\section{Consequences for Gravitino Dark Matter}

Local extensions of the supersymmetric standard model predict the existence of the gravitino, the supersymmetric partner of the graviton. Depending on the mechanism of supersymmetry breaking, it can be the lightest superparticle (LSP). In this case, it provides a natural dark matter candidate [85], and is an interesting alternative to the standard WIMP scenario.

The dominant contribution to the gravitino production in the early Universe comes from the 2-to-2 QCD scatterings, which are taking place in the thermal bath at reheating. The resulting energy density is given by

$$
\Omega_{3 / 2}^{\mathrm{th}} h^{2}=C\left(\frac{100 \mathrm{GeV}}{m_{3 / 2}}\right)\left(\frac{m_{\tilde{g}}}{1 \mathrm{TeV}}\right)^{2}\left(\frac{T_{R}}{10^{10} \mathrm{GeV}}\right),
$$

where $T_{R}$ is the reheating temperature, while $m_{3 / 2}$ and $m_{\tilde{g}}$ are the gravitino and gluino masses, respectively. To leading order in the gauge couplings, $C \simeq 0.5$ [86 88] 12 Remarkably, the observed dark matter relic density $\Omega_{\mathrm{DM}} h^{2}=0.11$ [89] is obtained for typical supersymmetric parameters, $m_{3 / 2} \sim 100 \mathrm{GeV}$ and $m_{\tilde{g}} \sim 1 \mathrm{TeV}$, and for reheating temperatures $T_{R} \sim 10^{10} \mathrm{TeV}$ as required for thermal leptogenesis to generate the observed baryon asymmetry [90, 91].

However, the above scenario is not free of problems: If $R$-parity is conserved, the decay of the next-to-lightest supersymmetric particle (NLSP) into gravitino and standard model particles is suppressed by the Planck scale. As a consequence, the NLSP becomes very long-lived and can dramatically affect the successful prediction of the standard big bang nucleosynthesis $(\mathrm{BBN})$ scenario [92 98]. Several alternative scenarios have been proposed to consistently accommodate thermal leptogenesis, gravitino dark matter and BBN, like assuming sneutrino [99] or stop [100] NLSPs, diluting the NLSP abundance by entropy production [101], or introducing new NLSP decay channels into hidden sector states [102, 103]. Also, the reheating temperature can be considerably reduced by considering nonthermal leptogenesis scenarios, as for example recently proposed in mechanisms where the universe is reheated through the decay of non-relativistic right-handed neutrinos produced in false vacuum decay [104, 105].

Alternatively, it has been proposed that $R$-parity could be mildly violated [30]. Such a violation could be e.g. produced by dynamical breaking of $\mathrm{U}(1)_{B-L}$ at low scales in the

\footnotetext{
${ }^{12}$ Note that $C$ has $\mathcal{O}(1)$ uncertainty due to unknown higher order contributions and nonperturbative effects [86].
} 
hidden sector [106]. It would lead to a rapid decay of the NLSP before the onset of BBN, thus rendering its impact on the standard cosmological picture negligible. Notably, even though the gravitino is not stable anymore in such a case, it still constitutes a viable dark matter candidate because its decay into standard model particles is doubly suppressed by the small $R$-parity breaking parameter as well as by the Planck mass [31], leading to lifetimes longer than the age of the Universe.

This $R$-parity violating scenario is particularly attractive because it opens up the possibility to probe gravitino dark matter by searching for its decay products in the cosmic-ray fluxes. In addition to cosmic anti-matter [35, 36] and neutrino [38] fluxes, the produced gamma-ray flux [30 34, 36] is of high interest because, besides the continuous component generated by the fragmentation of the Higgs and gauge bosons, the gamma-ray energy spectrum typically features an intense line, arising from the $\psi_{3 / 2} \rightarrow \gamma \nu$ two-body decay already at tree level.

In the rest of this section, we will apply the above Fermi LAT limits on gamma-ray lines to the decaying gravitino scenario. First, we will summarize the relevant aspects of bilinear $R$-parity violation, which will form the theoretical framework of our analysis. Second, we will present limits on the size of $R$-parity violation, and discuss prospect for seeing long-lived neutralino and stau NLSPs at the LHC, extending the analysis made in [107].

\section{$3.1 \quad R$-parity breaking model}

The supersymmetric standard model with bilinear $R$-parity breaking is, in addition to the standard $\mathrm{SU}(3)_{c} \times \mathrm{SU}(2)_{L} \times \mathrm{U}(1)_{Y}$ gauge interactions, defined by mass-mixing terms between lepton and Higgs fields in the superpotential

$$
W=W_{\mathrm{MSSM}}+\mu_{i} H_{u} l_{i}
$$

as well as by the mass-mixing terms in the scalar potential as induced by supersymmetry breaking

$$
\mathcal{L}=\mathcal{L}_{\text {soft }}^{\mathrm{MSSM}}+B_{i} H_{u} \tilde{l}_{i}+m_{i d}^{2} \tilde{l}_{i}^{\dagger} H_{d}+\text { h.c. } .
$$

Here, $W_{\text {MSSM }}$ and $\mathcal{L}_{\text {soft }}^{\text {MSSM }}$ are the usual $R$-parity conserving MSSM superpotential and scalar Lagrangian, $H_{u / d}$ are the up/down-type Higgs doublets, $l_{i}$ the lepton doublets, and $\mu_{i}, B_{i}$ and $m_{i d}^{2}$ are the $R$-parity violating couplings. As proposed in Ref. [107], it is convenient to work in a lepton-higgs basis where the mass mixings $\mu_{i}, B_{i}$ and $m_{i d}^{2}$ are traded for $R$-parity breaking Yukawa couplings. This can be achieved by a supersymmetric rotation of the superfields, followed by a non-supersymmetric rotation of the scalar fields alone. Besides the $R$-parity breaking Yukawa couplings, which will become relevant for the decay of the stau NLSP below, these rotations also generate mass-mixing terms between the neutralinos and the neutrinos. This neutrino-neutralino mixing, which can be parameterized by the dimensionless parameter $\zeta$, finally induces the gravitino decay mode $\psi_{3 / 2} \rightarrow \gamma \nu$ as 
well as the decay of neutralino NLSPs (see Ref. [107] for a definition of $\zeta$ in terms of the bilinear $R$-parity violating couplings $\mu_{i}, B_{i}$ and $\left.m_{i d}^{2}\right)$.

We will consider two typical sets of boundary conditions for the supersymmetry breaking parameters of the MSSM at the grand unification (GUT) scale for definiteness. The first one corresponds to equal scalar and gaugino masses

$$
\text { (A) } \quad m_{0}=m_{1 / 2}, \quad a_{0}=0, \quad \tan \beta=10,
$$

for which the bino-like neutralino $\chi_{1}^{0}$ is the NLSP. In the second one, which corresponds to no-scale models or gaugino mediation,

$$
\text { (B) } \quad m_{0}=0, \quad m_{1 / 2}, \quad a_{0}=0, \quad \tan \beta=10,
$$

the lightest stau $\tilde{\tau}_{1}$ is the NLSP. In both cases, $\tan \beta=10$ has been chosen as a representative value, and the trilinear scalar coupling $a_{0}$ has been set to zero for simplicity. The universal gaugino mass $m_{1 / 2}$ remains as the only independent variable. For both sets of boundary conditions, the gaugino masses $M_{1,2,3}$ satisfy the following relations at the electroweak scale

$$
\frac{M_{3}}{M_{1}} \simeq 5.9, \quad \frac{M_{2}}{M_{1}} \simeq 1.9
$$

Electroweak precision tests (EWPT) yield important lower bounds on the superparticle mass spectrum [88]. For a neutralino NLSP, the most stringent constraint comes from the Higgs potential. The universal gaugino mass $m_{1 / 2}$ is required to be high enough in order for the Higgs mass to fulfills the LEP lower bound $m_{h}>114.4 \mathrm{GeV}$ [108]. This implies the lower limit $m_{\chi_{1}^{0}} \gtrsim 130 \mathrm{GeV} 13$ However, allowing negative $a_{0}$ or scalar masses much larger than $m_{1 / 2}$ at the GUT scale would weaken this limit, and we will take $m_{\chi_{1}^{0}}>100 \mathrm{GeV}$ as a lower bound for the neutralino mass subsequently. In the stau NLSP case, the lower bound comes from the absence of pair production of heavy charged particles at LEP and reads $m_{\tilde{\tau}_{1}}>100 \mathrm{GeV}$ [108].

For a typical effective neutrino mass $\widetilde{m}_{1}=10^{-3} \mathrm{eV}$, successful thermal leptogenesis requires a minimal reheating temperature of $T_{R} \sim 10^{9} \mathrm{GeV}$ [91]. Using Eq. (11) together with a lower bound on the gluino mass $m_{\tilde{g}} \gtrsim 500 \mathrm{GeV}$ [109], this implies a lower bound for the gravitino mass $m_{3 / 2} \gtrsim 5 \mathrm{GeV}$.

In addition to the lower limits, NLSP mass upper limits follow from the requirement that the gravitino does not overclose the Universe. Indeed by rewriting Eq. (11) one obtains the constraint

$$
m_{\mathrm{NLSP}} \simeq 310 \mathrm{GeV}\left(\frac{\xi}{0.2}\right)\left(\frac{m_{3 / 2}}{100 \mathrm{GeV}}\right)^{1 / 2}\left(\frac{10^{9} \mathrm{GeV}}{T_{R}}\right)^{1 / 2}
$$

\footnotetext{
${ }^{13}$ Note that $m_{\chi_{1}^{0}} \simeq M_{1}$ with good accuracy [107].
} 
where $\xi \equiv m_{\mathrm{NLSP}} / m_{\tilde{g}}$ is implicitly fixed by the supersymmetry breaking boundary conditions [88]. Since $m_{\mathrm{NLSP}} \propto m_{3 / 2}^{1 / 2}$, requiring the gravitino to be the LSP induces absolute upper bounds on the NLSP masses. In the case of the neutralino NLSP, Eq. (17) implies $m_{\chi_{1}^{0}} \lesssim 690 \mathrm{GeV}$ for $\xi=1 / 5.9$, and is essentially independent of $m_{0}$ and $\tan \beta$. For the stau NLSP, $\tan \beta=10$ yields $\xi=1 / 6.2$, which consequently leads to the more stringent bounds $m_{\tilde{\tau}_{1}} \lesssim 615 \mathrm{GeV}$. Note that there is a strong dependence on $\tan \beta$ in that case [88], and that $\xi$ decreases with increasing $\tan \beta$.

\subsection{Fermi LAT constraints on the NLSP decay length}

The gravitino decay mode relevant for the discussion in this paper is the decay into photons and neutrinos via $\psi_{3 / 2} \rightarrow \gamma \nu$. However, decays into $W^{ \pm} \ell^{ \pm}, Z^{0} \nu$ and $h^{0} \nu$ final states are also possible, and have often large branching ratios. Below the corresponding kinematic thresholds, three-body decay with intermediate massive gauge bosons can become important in some cases [42].

Additionally to the gamma-ray line from $\psi_{3 / 2} \rightarrow \gamma \nu$, the different decay modes will produce a gamma-ray continuum, coming from Higgs and gauge boson fragmentation as well as from final state radiation of the charged leptons. This continuous spectrum has a cutoff at the position of the gamma-ray line, namely at half of the gravitino mass. Since we required in the above line search that all gamma-ray fluxes except the line follow a powerlaw locally around the line's energy ( $c f$. section 2.1), a too large continuum contribution from gravitino decay would render our line search strategy invalid. We expect this to become relevant for branching ratios into gamma-ray lines below $10^{-3} \ldots 10^{-2}$, and postpone a detailed analysis of this effect to future work. Using the branching ratios presented in Refs. [36, 38], we find that this problem can become severe for gravitino masses above a few hundred $\mathrm{GeV}, m_{3 / 2} \gtrsim 300 \mathrm{GeV}$. However, one has to keep in mind that the exact branching ratio into lines is in principle model-dependent. In what follows we assume for simplicity that no continuum contribution disturbs our line search, and we present limits over the full range of accessible gravitino masses.

The gravitino inverse decay rate into photon/neutrino pairs is given by 14 [31, 107]

$$
\Gamma_{\psi_{3 / 2} \rightarrow \gamma \nu}^{-1}=\frac{32 \sqrt{2}}{\alpha \zeta^{2}} \frac{G_{\mathrm{F}} M_{\mathrm{P}}^{2}}{m_{3 / 2}^{3}} \frac{M_{1}^{2} M_{2}^{2}}{\left(M_{2}-M_{1}\right)^{2}}\left(1+\mathcal{O}\left(s_{2 \beta} \frac{m_{Z}^{2}}{\mu^{2}}\right)\right)
$$

where $\alpha$ is the electromagnetic fine structure constant, $M_{\mathrm{P}}=2.4 \times 10^{18} \mathrm{GeV}$ the reduced Planck mass, and $G_{\mathrm{F}}=1.16 \times 10^{-5} \mathrm{GeV}^{-2}$ is the Fermi constant. Using this expression, the limits on the gamma-ray line flux can be translated into upper limits on the $R$-parity breaking parameter $\zeta$. A conservative bound on $\zeta$ is obtained for a given gravitino mass when using the corresponding maximally allowed bino mass. The latter is obtained by combining Eq. (11) and (16) considering the lowest reheating temperatures allowed in the thermal leptogenesis scenario, i.e. $T_{R} \sim 10^{9} \mathrm{GeV}$. The limits on the $R$-parity violation

\footnotetext{
${ }^{14}$ The correction factor is less than $10 \%$ for bino masses under consideration [107].
} 


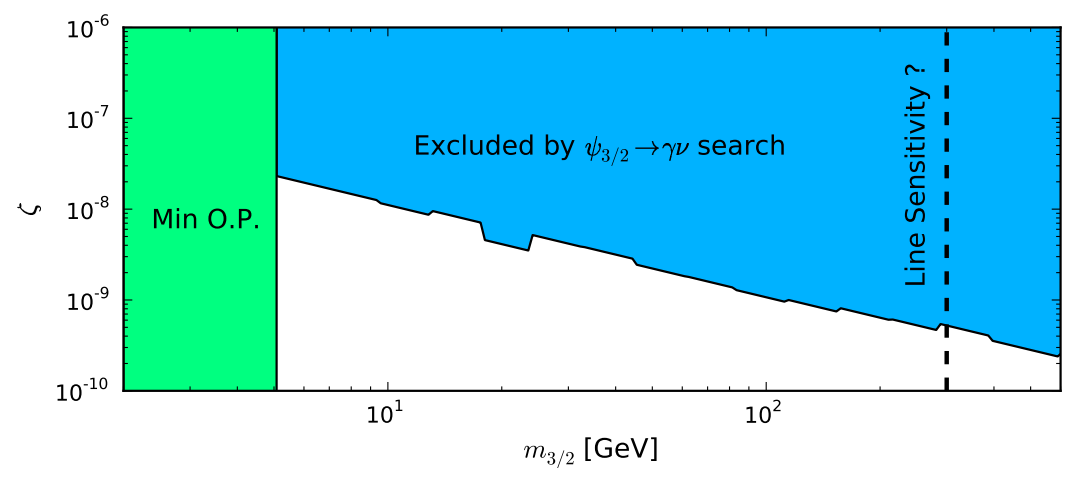

Figure 8: Upper bounds on the $R$-parity violation parameter $\zeta$, derived from the Fermi LAT gamma-ray line limits in Fig. 4. For thermal leptogenesis, overproduction (O.P.) of gravitinos already excludes the left green region. As discussed in the text, the adopted strategy for line searches might fail for gravitino masses above a few hundred GeV (dashed black line).

parameter $\zeta$ are presented in Fig. 8. As discussed above, for gravitino masses above a few hundred $\mathrm{GeV}$, our line search may fail due to a too large gamma-ray continuum contribution, which is indicated by the dashed line. Note also that, at these high gravitino masses, the production of anti-protons in gauge boson fragmentation could further constrain the $\zeta$ parameter [35, 36]. The implications of our gamma-ray line limits on the decay of the NLSP are discussed next.

\subsubsection{Stau NLSP}

In the case of the $\tilde{\tau}_{1}$-NLSP, the total decay width of the lightest mass eigenstate is a mixture of left and right handed partial decays

$$
\Gamma_{\tilde{\tau}_{1}}=\sin ^{2} \theta_{\tilde{\tau}} \Gamma_{\tilde{\tau}_{L}}+\cos ^{2} \theta_{\tilde{\tau}} \Gamma_{\tilde{\tau}_{R}} .
$$

Since the $R$-parity breaking Yukawa couplings are typically proportional to the ordinary Yukawa couplings, decays into second and third families dominate. For definiteness, we will below assume a flavor structure as described in Ref. [107], which is based on a FroggattNielsen U(1) flavor symmetry. In such a framework, the chiral state decays are dominated by the following channels [107]

$$
\begin{aligned}
& \tilde{\tau}_{R} \rightarrow \tau_{L} \nu, \mu_{L} \nu, \\
& \tilde{\tau}_{L} \rightarrow \bar{t}_{R} b_{L} .
\end{aligned}
$$

The corresponding decay rates are

$$
\begin{aligned}
\Gamma_{\tilde{\tau}_{L}} & =\frac{\epsilon^{2}}{16 \pi v^{2}} \frac{m_{t}^{2}}{m_{\tilde{\tau}_{1}}^{3}} 3\left(m_{\tilde{\tau}_{1}}^{2}-m_{t}^{2}-m_{b}^{2}\right) \sqrt{\left[m_{\tilde{\tau}_{1}}^{2}-\left(m_{t}+m_{b}\right)^{2}\right]\left[m_{\tilde{\tau}_{1}}^{2}-\left(m_{t}-m_{b}\right)^{2}\right]}, \\
\Gamma_{\tilde{\tau}_{R}} & =\frac{\epsilon^{2}}{16 \pi v^{2}} \frac{m_{t}^{2}}{m_{\tilde{\tau}_{1}}^{3}}(\tan \beta)^{2}\left(m_{\tilde{\tau}_{1}}^{2}-m_{\tau}^{2}\right)^{2},
\end{aligned}
$$




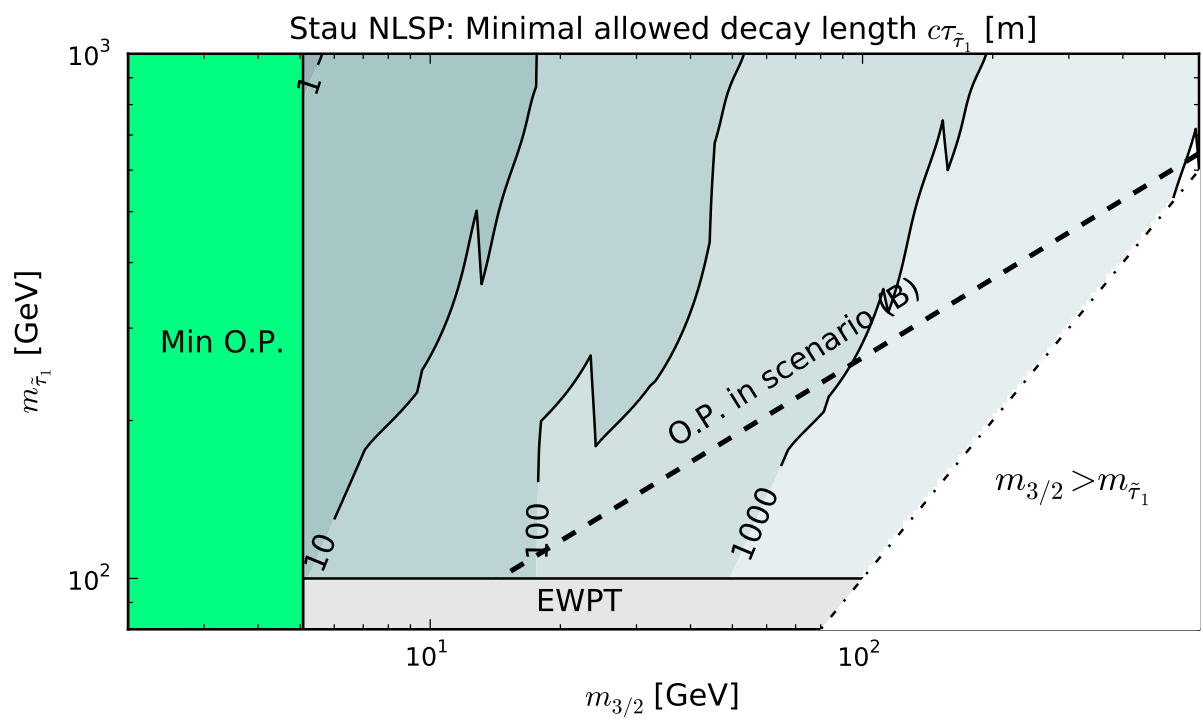

Figure 9: Contour plot of lower bounds on the stau NLSP decay length coming from the gamma-ray line constraints on the gravitino lifetime, as function of the stau and gravitino masses, $m_{\tilde{\tau}_{1}}$ and $m_{3 / 2}$ respectively. The lower gray region is excluded by electroweak precision tests (EWPT). For thermal leptogenesis, overproduction (O.P.) of gravitinos excludes at minimum the left green region, a limit which strengthens to the black-dashed line when assuming the universal boundary conditions (B), cf. Eq. (15). The lower-right exclusion comes from the gravitino LSP requirement. Note that for high gravitino masses above a few hundred GeV our adopted line search strategy might overestimate the limits on the NLSP decay length, because the branching ratio into gamma-ray lines can become very small (see text).

where the dimensionless parameter $\epsilon$ is directly related to the $R$-parity violating Yukawa couplings (see Ref. [107] for details). In principle $\zeta$ and $\epsilon$ are independent parameters because they stem from different linear combinations of the bilinear $R$-parity violating couplings $\mu_{i}, B_{i}$ and $m_{i d}^{2}$. We choose $\zeta \simeq \epsilon$ in order to set limits on the stau decay length. However, one has to keep in mind that in principle $\zeta$ could be set to values much smaller than $\epsilon$ by a proper choice of the parameters $\mu_{i}, B_{i}$ and $m_{i d}^{2}$. The behavior of the mixing angle $\theta_{\tilde{\tau}}$ with the stau mass $m_{\tilde{\tau}_{1}}$ can be deduced from the RGEs using the boundary conditions Eq. (15).

Using the upper limits on the $R$-parity breaking parameter $\zeta$ from Fig. 8 , we can derive lower bounds on the stau decay length. Our results are shown in Fig. 9, The parameter space is already constrained by EWPT and overproduction bounds, and the lower limits on the neutralino decay length vary between $100 \mathrm{~m}$ and $10 \mathrm{~km}$. It is interesting that if such particle were to be produced at the LHC, a sizable amount of their decay could take place in the detector [37]. We obtain a lowest possible decay length $c \tau_{\tilde{\tau}} \simeq 85 \mathrm{~m}$ for $m_{3 / 2} \simeq 16 \mathrm{GeV}$ and $m_{\tilde{\tau}_{1}} \simeq 100 \mathrm{GeV}$. 


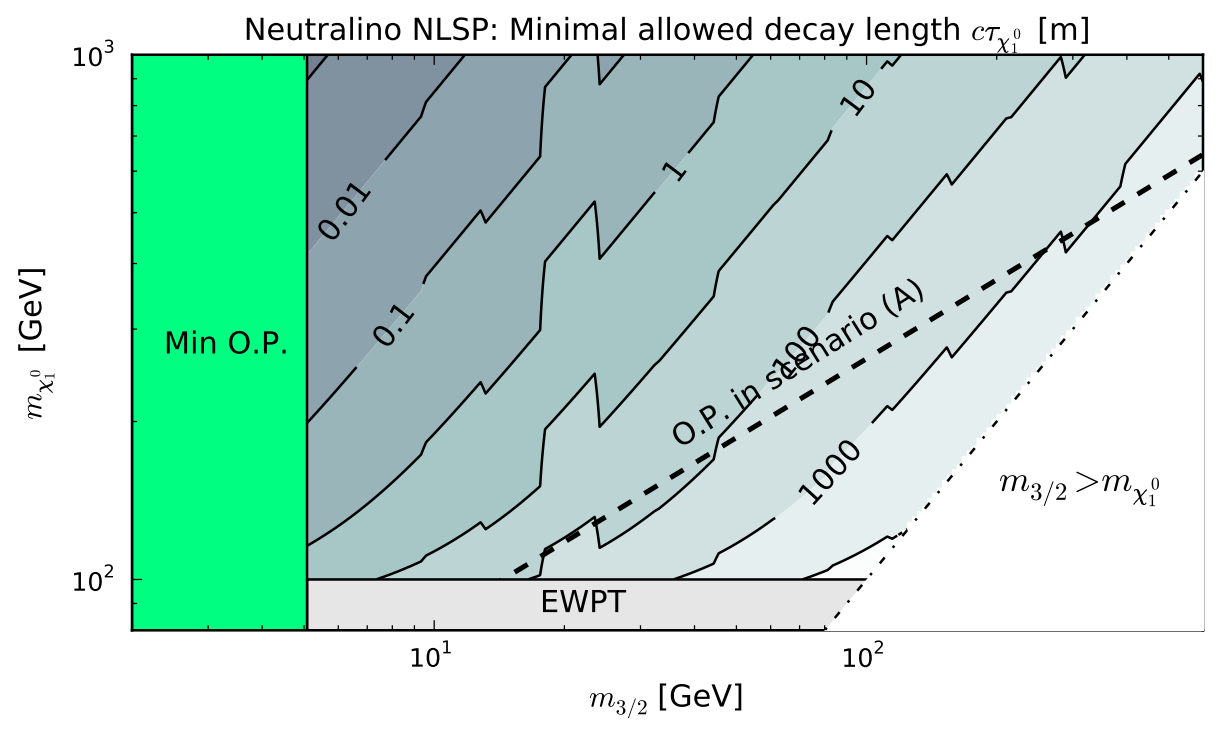

Figure 10: Like Fig. 9, but for a neutralino NLSP.

\subsubsection{Neutralino NLSP}

A neutralino NLSP heavier than $100 \mathrm{GeV}$ dominantly decays into $W^{ \pm} \ell^{\mp}$ and $Z^{0} \nu[110,111]$. The corresponding decay width is directly proportional to the $R$-parity breaking parameter $\zeta$ squared, which also enters the gravitino decay width in Eq. (18). As a consequence, the two quantities can be related through [107]

$$
\tau_{\chi_{1}^{0}}=\frac{c_{w}^{2}}{2 \sqrt{2}} \frac{\left(M_{2}-M_{1}\right)^{2}}{M_{2}^{2}} \frac{m_{3 / 2}^{3}}{G_{\mathrm{F}} M_{\mathrm{P}}^{2} m_{\chi_{1}^{0}}^{3}} \frac{\Gamma_{\psi_{3 / 2} \rightarrow \gamma \nu}^{-1}}{2 f\left(m_{\chi_{1}^{0}}, m_{W}\right)+f\left(m_{\chi_{1}^{0}}, m_{Z}\right)},
$$

where the phase space factor $f$ is defined by

$$
f\left(m_{1}, m_{2}\right)=\left(1-\frac{m_{2}^{2}}{m_{1}^{2}}\right)^{2}\left(1+2 \frac{m_{2}^{2}}{m_{1}^{2}}\right) .
$$

Using the gaugino mass relation from Eq. (16), lower bounds on the neutralino decay length $c \tau_{\chi_{1}^{0}}$ can then be derived from Tab. 3 and Fig. [4. Our results are summarized in Fig. 10. For the parameter space allowed by EWPT and overproduction bounds, we obtain minimal decay lengths $\mathcal{O}(100 \mathrm{~m}-10 \mathrm{~km})$, which are in the range of detectability of the LHC [37]. Decay lengths as small as $c \tau_{\chi_{1}^{0}} \simeq 50 \mathrm{~m}$ are allowed for $m_{3 / 2} \simeq 17 \mathrm{GeV}$ et $m_{\chi_{1}^{0}} \simeq 110 \mathrm{GeV}$.

\section{Conclusions}

We have presented a dedicated search for gamma-ray lines with energies between 1 and $300 \mathrm{GeV}$ in the diffuse gamma-ray fluxes measured by Fermi LAT between 4 Aug 2008 and 
17 Nov 2010, based on the public 'DataClean' event class. Such lines could be produced in dark matter annihilation or decay, and they are highly relevant for dark matter searches since they provide an essentially background free signature. To calculate the significance of potential gamma-ray lines in the diffuse flux, we used a binned profile likelihood method and fitted the observed energy spectrum locally with a line signal as expected from decaying or annihilating dark matter inside the Galactic halo plus a power law that resembles the astrophysical background fluxes. No gamma-ray line was found at $5 \sigma$ confidence level, and we derived conservative upper limits on the gamma-ray line flux that comes from regions with high Galactic latitudes or from the Galactic center (Tab. 3). We also discussed the apparent presence of several line-like features at the few percent level in the public data, which appear at energies below $20 \mathrm{GeV}$ with $\sim 3 \sigma$ significance and which are most probably due to instrumental effects.

Our conservative upper limits on the partial annihilation cross section of WIMP dark matter into $\gamma$-pairs, $\langle\sigma v\rangle_{\psi \psi \rightarrow \gamma \gamma}$, improve the EGRET limits in the range $E_{\gamma}=1-10 \mathrm{GeV}$ by up to an order of magnitude, while the previous Fermi LAT results $\left(E_{\gamma}=30-200 \mathrm{GeV}\right)$ are updated with two years of data and extended to the larger energy range $E_{\gamma}=1-300 \mathrm{GeV}$ (Fig. 5). Despite the increased statistics and the choice of a sky-region with optimized signal-to-noise ratio, the previous Fermi LAT limits are however only slightly improved. The reason is a somewhat worse energy resolution of the public data with respect to the dedicated Fermi LAT analysis as well as our strategy to always quote conservative limits in certain energy bands. The lower bounds on the inverse decay widths of dark matter into monochromatic photons, $\Gamma_{\psi \rightarrow \gamma \nu}^{-1}$, are at the level of $6 \times 10^{28} \mathrm{~s}$ over most of the considered energy regime (Fig. 4). Our results also constrain annihilation and decay channels with $\gamma Z^{0}$ final states.

In a $R$-parity breaking supersymmetric framework with gravitino LSP, we used the results of our gamma-ray line search to derive lower bounds on the gravitino lifetime. Considering supergravity models with two different types of universal boundary conditions at the grand unification scale, these lifetime constraints were used to set lower limits on the corresponding NLSP decay lengths. For gravitino and NLSP masses compatible with electroweak precision tests and overproduction constraints, we obtain $\mathcal{O}(100 \mathrm{~m}-10 \mathrm{~km})$ lower bounds on the NLSPs decay lengths. Interestingly, all these decay lengths would be accessible at the LHC.

Note. During the final stages of this work, first LHC limits on the CMSSM parameter space were published by the CMS collaboration [112], which will further constrain the superparticle masses discussed in this paper. Furthermore, the anonymous referee pointed us to a talk about the ongoing gamma-ray line analysis by the Fermi LAT collaboration, where preliminary results are presented that are, where they overlap, in agreement with our findings, and where line-like artefacts at lower energies in the current public data are mentioned 15

\footnotetext{
${ }^{15}$ See talk by E. Bloom at Aspen Winter Workshop on Indirect and Direct Detection of Dark Matter, Feb 2011, http://www.slac.stanford.edu/exp/glast/aspen11/talks.asp
} 


\section{Acknowledgements}

We thank Sergei Bobrovskyi, Wilfried Buchmüller, Jan Hajer and Alejandro Ibarra for useful discussions, David Paneque and Jeremy S. Perkins for helpful information on the Fermi LAT data, and Anthony R. Pullen for information about the EGRET analysis.

\section{References}

[1] G. Jungman, M. Kamionkowski, and K. Griest, Supersymmetric dark matter, Phys. Rept. 267 (1996) 195-373, hep-ph/9506380.

[2] L. Bergstrom, Non-baryonic dark matter: Observational evidence and detection methods, Rept. Prog. Phys. 63 (2000) 793, hep-ph/0002126.

[3] G. Bertone, D. Hooper, and J. Silk, Particle dark matter: Evidence, candidates and constraints, Phys. Rept. 405 (2005) 279-390, hep-ph/0404175.

[4] M. Srednicki, S. Theisen, and J. Silk, Cosmic Quarkonium: A Probe of Dark Matter, Phys. Rev. Lett. 56 (1986) 263.

[5] S. Rudaz, Cosmic production of quarkonium?, Phys. Rev. Lett. 56 (1986) 2128.

[6] L. Bergstrom and H. Snellman, Observable monochromatic photons from cosmic photino annihilation, Phys. Rev. D37 (1988) 3737-3741.

[7] S. P. Martin, A Supersymmetry Primer, hep-ph/9709356.

[8] S. Rudaz and F. W. Stecker, On the observability of the gamma-ray line flux from dark matter annihilation, Astrophys. J. 368 (1991) 406.

[9] L. Bergstrom, P. Ullio, and J. H. Buckley, Observability of gamma rays from dark matter neutralino annihilations in the Milky Way halo, Astropart. Phys. 9 (1998) 137-162, astro-ph/9712318.

[10] L. Bergstrom and P. Ullio, Full one-loop calculation of neutralino annihilation into two photons, Nucl. Phys. B504 (1997) 27-44, hep-ph/9706232.

[11] Z. Bern, P. Gondolo, and M. Perelstein, Neutralino annihilation into two photons, Phys. Lett. B411 (1997) 86-96, hep-ph/9706538.

[12] P. Ullio and L. Bergstrom, Neutralino annihilation into a photon and a $Z$ boson, Phys. Rev. D57 (1998) 1962-1971, hep-ph/9707333.

[13] P. Scott et. al., Direct Constraints on Minimal Supersymmetry from Fermi-LAT Observations of the Dwarf Galaxy Segue 1, JCAP 1001 (2010) 031, arXiv:0909.3300]. 
[14] J. Ripken, J. Conrad, and P. Scott, Direct constraints on the CMSSM using H.E.S.S. observations of the Galactic Centre and the Sagittarius dwarf galaxy, arXiv:1012.3939.

[15] S. Profumo, Hunting the lightest lightest neutralinos, Phys. Rev. D78 (2008) 023507, arXiv:0806.2150].

[16] F. Ferrer, L. M. Krauss, and S. Profumo, Indirect detection of light neutralino dark matter in the NMSSM, Phys. Rev. D74 (2006) 115007, hep-ph/0609257.

[17] S. Profumo, L. Ubaldi, and C. Wainwright, Singlet Scalar Dark Matter: monochromatic gamma rays and metastable vacua, Phys. Rev. D82 (2010) 123514, [arXiv:1009.5377.

[18] E. Dudas, Y. Mambrini, S. Pokorski, and A. Romagnoni, (In)visible Z' and dark matter, JHEP 08 (2009) 014, [arXiv:0904.1745].

[19] Y. Mambrini, A clear Dark Matter gamma ray line generated by the GreenSchwarz mechanism, JCAP 0912 (2009) 005, [arXiv:0907.2918].

[20] Y. Mambrini, Specific Dark Matter signatures from hidden U(1), arXiv:1012.0447.

[21] J. Goodman et. al., Gamma Ray Line Constraints on Effective Theories of Dark Matter, Nucl. Phys. B844 (2011) 55-68, [arXiv:1009.0008].

[22] C. B. Jackson, G. Servant, G. Shaughnessy, T. M. P. Tait, and M. Taoso, Higgs in Space!, JCAP 1004 (2010) 004, [arXiv:0912.0004].

[23] M. Gustafsson, E. Lundstrom, L. Bergstrom, and J. Edsjo, Significant gamma lines from inert Higgs dark matter, Phys. Rev. Lett. 99 (2007) 041301, astro-ph/0703512.

[24] G. Bertone, C. B. Jackson, G. Shaughnessy, T. M. P. Tait, and A. Vallinotto, The WIMP Forest: Indirect Detection of a Chiral Square, Phys. Rev. D80 (2009) 023512, [arXiv:0904.1442].

[25] G. Bertone, C. B. Jackson, G. Shaughnessy, T. M. P. Tait, and A. Vallinotto, Gamma Ray Lines from a Universal Extra Dimension, arXiv:1009.5107.

[26] L. Bergstrom, T. Bringmann, M. Eriksson, and M. Gustafsson, Gamma rays from heavy neutralino dark matter, Phys. Rev. Lett. 95 (2005) 241301, hep-ph/0507229.

[27] A. Birkedal, K. T. Matchev, M. Perelstein, and A. Spray, Robust gamma ray signature of WIMP dark matter, hep-ph/0507194.

[28] T. Bringmann, L. Bergstrom, and J. Edsjo, New Gamma-Ray Contributions to Supersymmetric Dark Matter Annihilation, JHEP 01 (2008) 049, [arXiv:0710.3169]. 
[29] L. Bergstrom, T. Bringmann, M. Eriksson, and M. Gustafsson, Gamma rays from Kaluza-Klein dark matter, Phys. Rev. Lett. 94 (2005) 131301, astro-ph/0410359.

[30] W. Buchmüller, L. Covi, K. Hamaguchi, A. Ibarra, and T. Yanagida, Gravitino dark matter in R-parity breaking vacua, JHEP 03 (2007) 037, hep-ph/0702184.

[31] F. Takayama and M. Yamaguchi, Gravitino dark matter without R-parity, Phys. Lett. B485 (2000) 388-392, hep-ph/0005214.

[32] G. Bertone, W. Buchmüller, L. Covi, and A. Ibarra, Gamma-Rays from Decaying Dark Matter, JCAP 0711 (2007) 003, arXiv:0709.2299].

[33] A. Ibarra and D. Tran, Gamma Ray Spectrum from Gravitino Dark Matter Decay, Phys. Rev. Lett. 100 (2008) 061301, [arXiv:0709.4593].

[34] S. Lola, P. Osland, and A. R. Raklev, Radiative gravitino decays from R-parity violation, Phys. Lett. B656 (2007) 83-90, [arXiv:0707.2510].

[35] A. Ibarra and D. Tran, Antimatter Signatures of Gravitino Dark Matter Decay, JCAP 0807 (2008) 002, [arXiv:0804.4596].

[36] K. Ishiwata, S. Matsumoto, and T. Moroi, High Energy Cosmic Rays from the Decay of Gravitino Dark Matter, Phys. Rev. D78 (2008) 063505, [arXiv:0805.1133].

[37] K. Ishiwata, T. Ito, and T. Moroi, Long-Lived Unstable Superparticles at the LHC, Phys. Lett. B669 (2008) 28-33, [arXiv:0807.0975].

[38] L. Covi, M. Grefe, A. Ibarra, and D. Tran, Unstable Gravitino Dark Matter and Neutrino Flux, JCAP 0901 (2009) 029, [arXiv:0809.5030].

[39] W. Buchmüller, A. Ibarra, T. Shindou, F. Takayama, and D. Tran, Probing Gravitino Dark Matter, JCAP 0909 (2009) 021, [arXiv:0906.1187].

[40] K.-Y. Choi, D. E. Lopez-Fogliani, C. Munoz, and R. R. de Austri, Gamma-ray detection from gravitino dark matter decay in the $\mu \nu S S M, J C A P 1003$ (2010) 028, [arXiv:0906.3681].

[41] N. E. Bomark, S. Lola, P. Osland, and A. R. Raklev, Photon, Neutrino and Charged Particle Spectra from R-violating Gravitino Decays, Phys. Lett. B686 (2010) 152-161, [arXiv:0911.3376].

[42] K.-Y. Choi and C. E. Yaguna, New decay modes of gravitino dark matter, Phys. Rev. D82 (2010) 015008, [arXiv:1003.3401].

[43] K.-Y. Choi, D. Restrepo, C. E. Yaguna, and O. Zapata, Indirect detection of gravitino dark matter including its three-body decays, JCAP 1010 (2010) 033, [arXiv:1007.1728]. 
[44] D. Eichler, TeV particles as weakly unstable dark matter, Phys. Rev. Lett. 63 (1989) 2440 .

[45] A. Arvanitaki et. al., Decaying Dark Matter as a Probe of Unification and TeV Spectroscopy, Phys. Rev. D80 (2009) 055011, [arXiv:0904.2789].

[46] C. Arina, T. Hambye, A. Ibarra, and C. Weniger, Intense Gamma-Ray Lines from Hidden Vector Dark Matter Decay, JCAP 1003 (2010) 024, [arXiv:0912.4496].

[47] PAMELA Collaboration, O. Adriani et. al., An anomalous positron abundance in cosmic rays with energies 1.5-100 GeV, Nature 458 (2009) 607-609, arXiv:0810.4995].

[48] Fermi LAT Collaboration, A. A. Abdo et. al., Measurement of the Cosmic Ray e+ plus e-spectrum from $20 \mathrm{GeV}$ to 1 TeV with the Fermi Large Area Telescope, Phys. Rev. Lett. 102 (2009) 181101, [arXiv:0905.0025].

[49] Fermi LAT Collaboration, M. Ackermann et. al., Fermi LAT observations of cosmic-ray electrons from 7 GeV to 1 TeV, Phys. Rev. D82 (2010) 092004, [arXiv:1008.3999].

[50] M. Garny, A. Ibarra, D. Tran, and C. Weniger, Gamma-Ray Lines from Radiative Dark Matter Decay, arXiv:1011.3786.

[51] Fermi LAT Collaboration, W. B. Atwood et. al., The Large Area Telescope on the Fermi Gamma-ray Space Telescope Mission, Astrophys. J. 697 (2009) 1071-1102, [arXiv:0902.1089].

[52] A. A. Abdo et. al., Fermi LAT Search for Photon Lines from 30 to 200 GeV and Dark Matter Implications, Phys. Rev. Lett. 104 (2010) 091302, [arXiv:1001.4836].

[53] A. R. Pullen, R.-R. Chary, and M. Kamionkowski, Search with EGRET for a Gamma Ray Line from the Galactic Center, Phys.Rev. D76 (2007) 063006, astro-ph/0610295.

[54] Fermi LAT Collaboration, A. A. Abdo et. al., The Spectrum of the Isotropic Diffuse Gamma-Ray Emission Derived From First-Year Fermi Large Area Telescope Data, Phys. Rev. Lett. 104 (2010) 101101, [arXiv:1002.3603].

[55] Fermi LAT Collaboration, A. A. Abdo et. al., Constraints on Cosmological Dark Matter Annihilation from the Fermi-LAT Isotropic Diffuse Gamma-Ray Measurement, JCAP 1004 (2010) 014, [arXiv:1002.4415].

[56] L. Bergstrom, J. Edsjo, and P. Ullio, Spectral gamma-ray signatures of cosmological dark matter annihilations, Phys. Rev. Lett. 87 (2001) 251301, astro-ph/0105048. 
[57] P. Ullio, L. Bergstrom, J. Edsjo, and C. G. Lacey, Cosmological dark matter annihilations into gamma-rays: A closer look, Phys. Rev. D66 (2002) 123502, astro-ph/0207125.

[58] G. D. Mack, T. D. Jacques, J. F. Beacom, N. F. Bell, and H. Yuksel, Conservative Constraints on Dark Matter Annihilation into Gamma Rays, Phys.Rev. D78 (2008) 063542, arXiv:0803.0157.

[59] H. Yuksel and M. D. Kistler, Circumscribing late dark matter decays model independently, Phys.Rev. D78 (2008) 023502, [arXiv:0711.2906].

[60] P. D. Serpico and G. Zaharijas, Optimal angular window for observing Dark Matter annihilation from the Galactic Center region: the case of $\gamma$-ray lines, Astropart. Phys. 29 (2008) 380-385, arXiv:0802.3245.

[61] P. D. Serpico and D. Hooper, Gamma rays from Dark Matter Annihilation in the Central Region of the Galaxy, New J. Phys. 11 (2009) 105010, [arXiv:0902.2539].

[62] Z.-C. Tang, Q. Yuan, X.-J. Bi, and G.-M. Chen, On the Detectability of Galactic Dark Matter Annihilation into Monochromatic Gamma-rays, arXiv:1008.2046. * Temporary entry *

[63] The CDMS-II Collaboration, Z. Ahmed et. al., Dark Matter Search Results from the CDMS II Experiment, Science 327 (2010) 1619-1621, [arXiv:0912.3592].

[64] CoGeNT Collaboration, C. E. Aalseth et. al., Results from a Search for Light-Mass Dark Matter with a P-type Point Contact Germanium Detector, arXiv:1002.4703.

[65] DAMA Collaboration, R. Bernabei et. al., First results from DAMA/LIBRA and the combined results with DAMA/NaI, Eur. Phys. J. C56 (2008) 333-355, arXiv:0804.2741].

[66] R. Bernabei et. al., New results from DAMA/LIBRA, Eur. Phys. J. C67 (2010) 39-49, arXiv:1002.1028].

[67] A. M. Ghez et. al., Measuring Distance and Properties of the Milky Way's Central Supermassive Black Hole with Stellar Orbits, Astrophys. J. 689 (2008) 1044-1062, arXiv:0808.2870].

[68] S. Gillessen et. al., Monitoring stellar orbits around the Massive Black Hole in the Galactic Center, Astrophys. J. 692 (2009) 1075-1109, [arXiv:0810.4674].

[69] J. F. Navarro, C. S. Frenk, and S. D. White, A Universal density profile from hierarchical clustering, Astrophys.J. 490 (1997) 493-508, astro-ph/9611107. 
[70] J. F. Navarro et. al., The Inner Structure of LambdaCDM Halos III: Universality and Asymptotic Slopes, Mon. Not. Roy. Astron. Soc. 349 (2004) 1039, astro-ph/0311231.

[71] V. Springel et. al., The Aquarius Project: the subhalos of galactic halos, Mon. Not. Roy. Astron. Soc. 391 (2008) 1685-1711, [arXiv:0809.0898].

[72] L. Pieri, J. Lavalle, G. Bertone, and E. Branchini, Implications of High-Resolution Simulations on Indirect Dark Matter Searches, arXiv:0908.0195.

[73] P. Salucci et. al., The universal rotation curve of spiral galaxies. II: The dark matter distribution out to the virial radius, Mon. Not. Roy. Astron. Soc. 378 (2007) 41-47, astro-ph/0703115.

[74] P. Salucci, F. Nesti, G. Gentile, and C. F. Martins, The dark matter density at the Sun's location, Astron. Astrophys. 523 (2010) A83, [arXiv:1003.3101].

[75] R. Catena and P. Ullio, A novel determination of the local dark matter density, JCAP 1008 (2010) 004, [arXiv:0907.0018].

[76] Fermi LAT Collaboration, Y. Edmonds et. al., Estimate for GLAST LAT Milky Way dark matter WIMP line sensitivity, AIP Conf. Proc. 921 (2007) 514-515.

[77] http://fermi.gsfc.nasa.gov/ssc/data/analysis/LAT_caveats.html.

[78] R. Rando and f. t. F. L. Collaboration, Post-launch performance of the Fermi Large Area Telescope, arXiv:0907.0626.

[79] Fermi LAT Collaboration, A. A. Abdo et. al., The On-orbit Calibrations for the Fermi Large Area Telescope, Astropart. Phys. 32 (2009) 193-219, [arXiv:0904.2226].

[80] W. A. Rolke, A. M. Lopez, and J. Conrad, Confidence Intervals with Frequentist Treatment of Statistical and Systematic Uncertainties, Nucl. Instrum. Meth. A551 (2005) 493-503, physics/0403059.

[81] J. Conrad, T. Ylinen, and J. Scargle, Statistical analysis of detection of, and upper limits on, dark matter lines, AIP Conf. Proc. 921 (2007) 586-587.

[82] S. S. Wilks, The large-sample distribution of the likelihood ratio for testing composite hypotheses, The Annals of Mathematical Statistics 9 (1938) 60-62.

[83] G. J. Feldman and R. D. Cousins, A Unified Approach to the Classical Statistical Analysis of Small Signals, Phys. Rev. D57 (1998) 3873-3889, physics/9711021.

[84] Fermi LAT Collaboration, T. Ylinen, Annihilation lines from dark matter with the Fermi-LAT, AIP Conf. Proc. 1241 (2010) 463-467. 
[85] H. Pagels and J. R. Primack, Supersymmetry, Cosmology and New TeV Physics, Phys. Rev. Lett. 48 (1982) 223.

[86] M. Bolz, A. Brandenburg, and W. Buchmüller, Thermal Production of Gravitinos, Nucl. Phys. B606 (2001) 518-544, hep-ph/0012052.

[87] J. Pradler and F. D. Steffen, Thermal Gravitino Production and Collider Tests of Leptogenesis, Phys. Rev. D75 (2007) 023509, hep-ph/0608344.

[88] W. Buchmüller, M. Endo, and T. Shindou, Superparticle Mass Window from Leptogenesis and Decaying Gravitino Dark Matter, JHEP 11 (2008) 079, [arXiv:0809.4667].

[89] E. Komatsu et. al., Seven-Year Wilkinson Microwave Anisotropy Probe (WMAP) Observations: Cosmological Interpretation, arXiv:1001.4538.

[90] M. Fukugita and T. Yanagida, Baryogenesis Without Grand Unification, Phys. Lett. B174 (1986) 45.

[91] W. Buchmüller, P. Di Bari, and M. Plumacher, Leptogenesis for pedestrians, Ann. Phys. 315 (2005) 305-351, hep-ph/0401240].

[92] J. R. Ellis, D. V. Nanopoulos, and S. Sarkar, The Cosmology of Decaying Gravitinos, Nucl. Phys. B259 (1985) 175.

[93] S. Sarkar, Big bang nucleosynthesis and physics beyond the standard model, Rept. Prog. Phys. 59 (1996) 1493-1610, hep-ph/9602260.

[94] M. Kawasaki, K. Kohri, and T. Moroi, Big-bang nucleosynthesis and hadronic decay of long-lived massive particles, Phys. Rev. D71 (2005) 083502, astro-ph/0408426.

[95] M. Pospelov, Particle physics catalysis of thermal big bang nucleosynthesis, Phys. Rev. Lett. 98 (2007) 231301, hep-ph/0605215.

[96] K. Hamaguchi, T. Hatsuda, M. Kamimura, Y. Kino, and T. T. Yanagida, Stau-catalyzed Li-6 production in big-bang nucleosynthesis, Phys. Lett. B650 (2007) 268-274, hep-ph/0702274.

[97] M. Pospelov, J. Pradler, and F. D. Steffen, Constraints on Supersymmetric Models from Catalytic Primordial Nucleosynthesis of Beryllium, JCAP 0811 (2008) 020, [arXiv:0807.4287].

[98] M. Kawasaki, K. Kohri, T. Moroi, and A. Yotsuyanagi, Big-Bang Nucleosynthesis and Gravitino, Phys. Rev. D78 (2008) 065011, [arXiv:0804.3745].

[99] T. Kanzaki, M. Kawasaki, K. Kohri, and T. Moroi, Cosmological Constraints on Gravitino LSP Scenario with Sneutrino NLSP, Phys. Rev. D75 (2007) 025011, hep-ph/0609246. 
[100] J. L. Diaz-Cruz, J. R. Ellis, K. A. Olive, and Y. Santoso, On the feasibility of a stop NLSP in gravitino dark matter scenarios, JHEP 05 (2007) 003, hep-ph/0701229.

[101] J. Pradler and F. D. Steffen, Constraints on the reheating temperature in gravitino dark matter scenarios, Phys. Lett. B648 (2007) 224-235, hep-ph/0612291.

[102] A. De Simone, M. Garny, A. Ibarra, and C. Weniger, Supersymmetric Leptogenesis with a Light Hidden Sector, JCAP 1007 (2010) 017, [arXiv:1004.4890].

[103] C. Cheung, J. Mardon, Y. Nomura, and J. Thaler, A Definitive Signal of Multiple Supersymmetry Breaking, JHEP 07 (2010) 035, [arXiv:1004.4637].

[104] W. Buchmüller, K. Schmitz, and G. Vertongen, Matter and Dark Matter from False Vacuum Decay, Phys. Lett. B693 (2010) 421-425, [arXiv:1008.2355].

[105] W. Buchmuller, K. Schmitz, and G. Vertongen, Work in preparation, .

[106] J. Schmidt, C. Weniger, and T. T. Yanagida, Dynamical Matter-Parity Breaking and Gravitino Dark Matter, Phys. Rev. D82 (2010) 103517, [arXiv:1008.0398].

[107] S. Bobrovskyi, W. Buchmüller, J. Hajer, and J. Schmidt, Broken R-Parity in the Sky and at the LHC, JHEP 10 (2010) 061, [arXiv:1007.5007].

[108] Particle Data Group Collaboration, K. Nakamura et. al., Review of particle physics, J. Phys. G37 (2010) 075021.

[109] J. Alwall, M.-P. Le, M. Lisanti, and J. G. Wacker, Searching for Directly Decaying Gluinos at the Tevatron, Phys. Lett. B666 (2008) 34-37, [arXiv:0803.0019].

[110] B. Mukhopadhyaya, S. Roy, and F. Vissani, Correlation between neutrino oscillations and collider signals of supersymmetry in an $R$-parity violating model, Phys. Lett. B443 (1998) 191-195, hep-ph/9808265.

[111] E. J. Chun and J. S. Lee, Implication of Super-Kamiokande data on R-parity violation, Phys. Rev. D60 (1999) 075006, hep-ph/9811201.

[112] C. Collaboration, Search for Supersymmetry in pp Collisions at 7 Te V in Events with Jets and Missing Transverse Energy, arXiv:1101.1628. 